Revista de Biología Marina y Oceanografía

Vol. 48, №3: 565-584, diciembre 2013

DOI 10.4067/S0718-19572013000300013

Artículo

\title{
Zoogeografía de los bivalvos marinos de la costa de Tamaulipas, México
}

\author{
Marine bivalves zoogeography in the Tamaulipas coast, Mexico
}

Alfonso Correa-Sandoval ${ }^{1}$ y Jorge H. Rodríguez-Castro ${ }^{1}$

'División de Estudios de Posgrado e Investigación, Instituto Tecnológico de Ciudad Victoria, Boulevard Emilio Portes Gil 1301 Poniente, Apdo. Postal 175, C.P. 87010, Ciudad Victoria, Tamaulipas, México. agutierr@uat.edu.mx

\begin{abstract}
This study provides both a current taxonomic listing of known species of marine bivalves from the coast of Tamaulipas, Mexico as well as an overall assessment of their poorly known biogeographical relationships. Marine bivalves were surveyed (midlittoral, supralitoral and sublittoral zones) from September 1987 to April 2010. One hundred and thirtyone species of marine bivalves representing 98 genera in 37 families are reported. Two species are first records for Tamaulipas. These 131 species share the following malacogeographical relationships: 62 species (47.32\%) exhibit Caribbean and Carolinian affinities, 20 species (15.26\%) exhibit Carolinian and Caribbean affinities, 13 species (9.92\%) exhibit Carolinian and Argentinean affinities and 12 species (9.16\%) exhibit Caribbean, Carolinian and Boreal affinities. Only 6 species (4.58\%) exhibit an exclusive Caribbean distribution and 4 species (3.05\%) are known only from the Gulf of Mexico. The fact that $81.66 \%$ (107 species) of the marine Tamaulipan bivalve fauna exhibits the above malacogeographical relationships provides overwhelming evidence that the Tamaulipan coast of Mexico represents a transitional zone between the Carolinian and Caribbean malacological provinces.
\end{abstract}

Key words: Mollusks, biogeography, Caribbean and Carolinian provinces

Resumen.- La biogeografía de los bivalvos marinos mexicanos es pobremente conocida, aunque varios estudios se han realizado pero con un enfoque taxonómico. Este estudio contribuye con nueva información sobre la distribución geográfica de los bivalvos marinos de la costa del estado de Tamaulipas. Entre septiembre de 1987 y abril de 2010 se estudiaron los bivalvos marinos (zonas mesolitoral, supralitoral y sublitorial) del área de estudio. Se registran 131 especies incluidas en 98 géneros y 37 familias. Dos especies son primeros registros para el estado. Las principales afinidades malacogeográficas son la principalmente Caribeña y Caroliniana (62 especies: 47,32\%), Caroliniana y Caribeña (20 especies: 15,26\%), principalmente Caribeña, Caroliniana y Argentiniana (13 especies: 9,92\%) y la principalmente Caribeña, Caroliniana y Boreal con 12 especies (9,16\%) que juntas suman el 81,66\% del total de las especies (107 especies), lo que prueba que la costa tamaulipeca es una zona de transición de las provincias malacológicas Caroliniana y Caribeña. Sólo 6 especies $(4,58 \%)$ poseen una afinidad exclusivamente Caribeña. Cuatro especies $(3,05 \%)$ restringen su ámbito geográfico conocido al Golfo de México.

Palabras clave: Moluscos, biogeografía, provincias Caribeña y Caroliniana

\section{INTRODUCCIÓN}

Considerando la distribución geográfica de las especies y la pérdida de las mismas a escala global debido al efecto negativo de la actividad humana en los ecosistemas, el reconocimiento, análisis y comparación de los patrones biogeográficos es esencial para la conservación de la biodiversidad (Llorente \& Morrone 2001, Lomolino et al. 2010). En cuanto al estudio de los moluscos a nivel mundial, en los años recientes los bivalvos han recibido menos atención que los gastrópodos y cefalópodos (Thorpe 2006) y, en el caso de la fauna malacológica de la plataforma y talud continentales del Golfo de México y Mar Caribe los trabajos de autores mexicanos son relativamente recientes (Pérez-Rodríguez 1980, GarcíaCubas et al. 1999).

Varios estudios han realizado extensos reconocimientos taxonómicos de los bivalvos marinos del Golfo de México (Morris 1975, Abbott \& Morris 1995, Pérez-Rodríguez 1997, García-Cubas \& Reguero 2007, Turgeon et al. 2009) e indican además datos de distribución geográfica; Warmke \& Abbott (1961), señalan la afinidad Caribeña de algunas especies y Abbott (1996) describe las provincias malacológicas en el Atlántico americano. Sin embargo, son necesarias publicaciones que establezcan para la zona o para alguno de los 6 estados mexicanos colindantes 
con el Golfo de México, las afinidades de las especies de bivalvos marinos de acuerdo a su distribución geográfica, a las diversas provincias malacológicas como parte de la biogeografía descriptiva, y como elemento básico de la biogeografía cuantitativa, para contribuir a explicar su distribución.

Dado lo anterior, en este trabajo se exponen los resultados sobre los ámbitos de la distribución geográfica conocida en el Atlántico americano de las especies de bivalvos marinos de la costa de Tamaulipas, se establecen sus afinidades malacogeográficas y se analiza la distribución geográfica de algunas especies.

\section{Materiales Y MÉTODOS}

El área de estudio corresponde a la costa del estado de Tamaulipas $\left(25^{\circ} 56^{\prime}\right.$ y $22^{\circ} 14^{\prime} \mathrm{N}$ y $97^{\circ} 14^{\prime}$ y $97^{\circ} 48^{\prime} \mathrm{O}$, Fig. 1 ), la cual tiene una extensión aproximada de $420 \mathrm{~km}$. Las temperaturas promedio anuales varían de $25,5^{\circ} \mathrm{C}$ a $27,5^{\circ} \mathrm{C}$ y las salinidades de 30 a 35,2 (Britton \& Morton 1989, De La Lanza et al. 2004).
En este estudio se incluyeron las especies de 13 localidades de la costa tamaulipeca (Apéndice 1) visitadas una vez cada 2 años a partir de septiembre de 1987 y hasta abril de 2010. La duración del trabajo de campo en cada localidad fue de un día.

La recolección fue manual a lo largo de $3 \mathrm{~km}$ de la franja intermareal en cada localidad. También se revisó la zona supralitoral (alrededor de $20 \mathrm{~m}$ ), se hicieron inmersiones (una por localidad) con duración aproximada de $2 \mathrm{~h}$ hasta $2 \mathrm{~m}$ de profundidad, en transectos de 100 a $150 \mathrm{~m}$ paralelos a la playa. Como complemento se obtuvieron también ejemplares por arrastre camaronero (de $1 \mathrm{~h}$, a 3 nudos) uno por lo menos en las cercanías de cada localidad, a partir de septiembre de 2005. La embarcación que se usó poseía $30 \mathrm{~m}$ de eslora con red de arrastre para fondo blando. Se obtuvieron uno o varios organismos por especie y por localidad, los cuales se conservaron en alcohol isopropílico al $70 \%$. Los especímenes encontrados en conchales de la playa se conservaron en seco. Se revisaron en total 589 lotes de bivalvos (4.334 ejemplares).

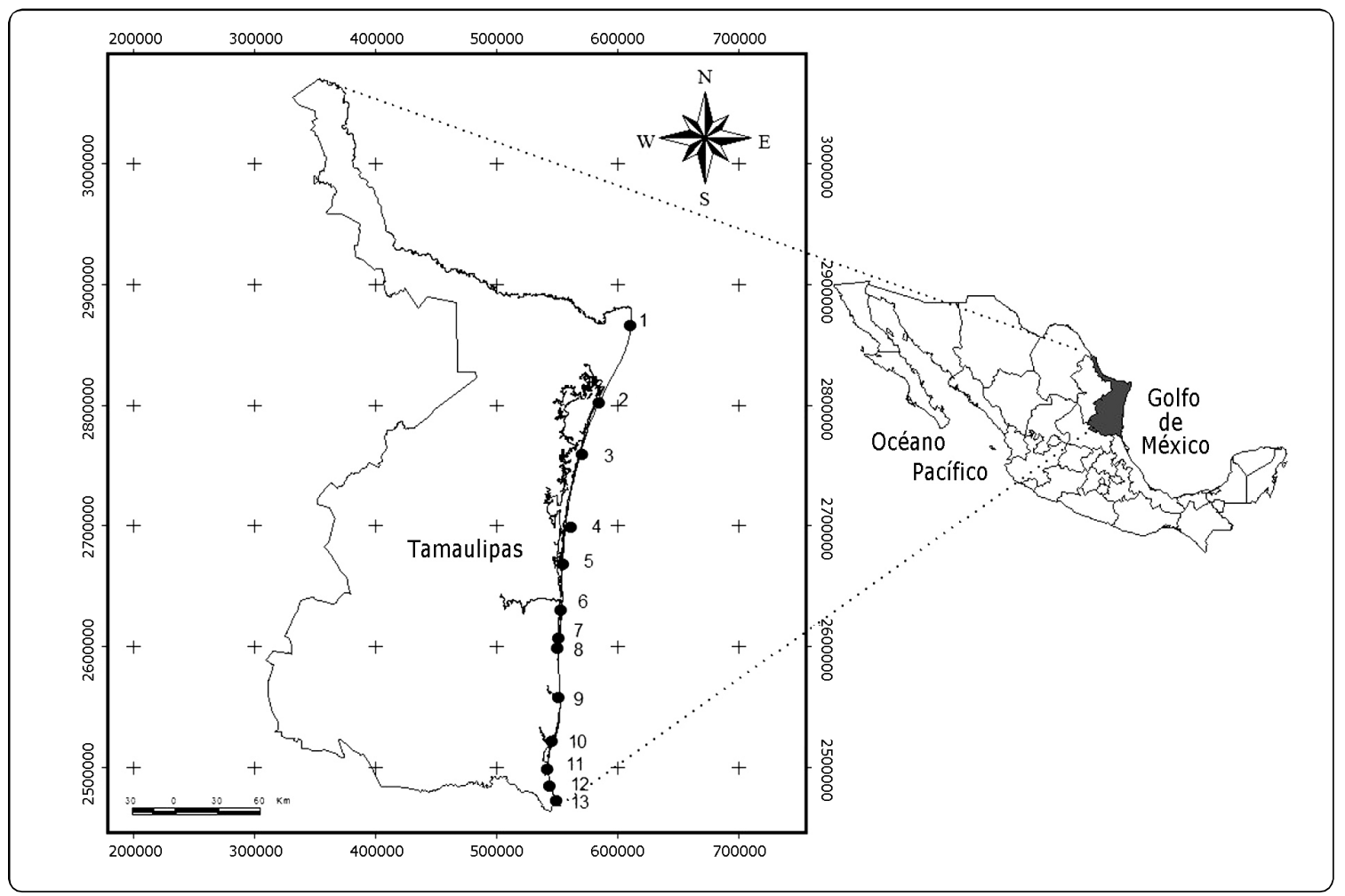

Figura 1. Localidades de muestreo en la costa del Estado de Tamaulipas (Ver Apéndice 1) / Localities sampled in the coast of Tamaulipas (See Appendix 1) 
El material determinado y etiquetado se depositó en la Colección Malacológica del Instituto Tecnológico de Ciudad Victoria.

Se complementó la lista de especies para el área de estudio con los trabajos de Abbott (1974, 1996), Morris (1975), Leal (1978), Vokes \& Vokes (1983), Andrews (1992), Abbott \& Morris (1995), Pérez-Rodríguez (1997), Rehder (2000), Redfern (2001), García-Cubas \& Reguero (2007) y Turgeon et al. (2009), entre los principales.

En el Apéndice 2 el arreglo de las familias y la actualización de los nombres científicos, con la consideración de las sinonimias en su caso, siguió la propuesta de Mikkelsen \& Bieler (2008), Turgeon et al. (2009), Tunnell et al. (2010) y Coan \& Valentich-Scott (2012).

La presencia de las especies de bivalvos marinos en las localidades del área de estudio se indica en el Apéndice 2. La revisión de la distribución geográfica específica en otras áreas del Golfo de México y la presencia en otras regiones del Atlántico americano fue de acuerdo con los trabajos de Warmke \& Abbott (1961), Rice \& Kornicker (1962), Rice (1965), Abbott (1970, 1974, 1996), Eckdale (1974), Morris (1975), Abbott \& Zim (1978), PérezRodríguez (1980, 1997), García-Cubas (1981), Andrews (1992), Reguero (1994), Abbott \& Morris (1995), Shelton (1997), Abbott \& Dance (2000), Rehder (2000), Redfern (2001), Turgeon et al. (2009), Tunnell et al. (2010).

Para establecer la afinidad malacogeográfica de las especies se combinaron las provincias malacológicas propuestas por Warmke \& Abbott (1961), Abbott \& Zim (1978), Sabelli (1980), Abbott (1996) y Petuch (2013).

El área geográfica considerada para definir las especies restringidas al Golfo de México fue del sur de Florida a las costas del Caribe mexicano.

\section{Resultados}

Las 131 especies de bivalvos marinos de la costa de Tamaulipas se distribuyen en 37 familias (Apéndice 2).

Se identificaron 9 afinidades malacogeográficas en los bivalvos marinos de la costa de Tamaulipas (Apéndice 2). La más importante es la principalmente Caribeña y Caroliniana con 61 especies (47,32\% de esta malacofauna). Le siguen en importancia las afinidades Caroliniana y Caribeña con 20 especies $(15,26 \%)$, la principalmente Caribeña, Caroliniana y Argentiniana con 13 especies $(9,92 \%)$ y la principalmente Caribeña, Caroliniana y Boreal con 12 especies $(9,16 \%)$. Las 4 categorías anteriores suman el $81,66 \%$ de esta malacofauna.

Sólo 6 especies $(4,58 \%)$ presentaron una afinidad exclusivamente Caribeña: Argopecten nucleus, Chama sinuosa, Cardita gracilis, Papyridea semisulcata, Asaphis deflorata y Lyrophora paphia.

La afinidad principalmente Caroliniana y Caribeña presentó sólo una especie $(0,76 \%)$.

Anatina anatina y Macrocallista nimbosa se registran por primera vez para el estado de Tamaulipas.

Aligena texasiana, Rangianella flexuosa, Eurytellina alternata tayloriana y Lyrophora clenchi restringen su distribución geográfica conocida al Golfo de México.

\section{Discusión}

Todas las especies de bivalvos marinos de la costa de Tamaulipas presentan en algún grado la afinidad Caribeña en su distribución geográfica. Ninguna de las especies presentes en el área ha sido exclusivamente Caroliniana.

Seis especies de bivalvos marinos no tienen presencia conocida en la provincia Caroliniana. Es decir, son exclusivamente Caribeñas.

Sin embargo, 8 de las 9 afinidades malacogeográficas (Tabla 1) consideran tanto a la Caribeña como a la Caroliniana, y las 4 principales afinidades suman el $81,66 \%$. Al adicionar la siguiente afinidad con más especies (Boreal, Caroliniana y Caribeña: 11 especies) el valor se incrementa a 90,06\% (118 especies), lo que indica que la costa de Tamaulipas es una zona de transición de las extensas provincias malacológicas Caroliniana y Caribeña.

Llama la atención la distribución no uniforme de algunas especies como Semele purpurascens señalada en Tabasco y Campeche por Pérez-Rodríguez (1997), con presencia al norte del Golfo de México en Alabama y Texas (Pulley 1952, Tunnell \& Chaney 1970, Turgeon et al. 2009) y también hallada en este estudio en Tamaulipas (Tepehuajes). Dados los estudios realizados en Veracruz (Reguero 1994, García-Cubas et al. 1994) es muy probable el carácter discontinuo de la distribución de esta especie que es señalada por Pérez-Rodríguez (1980) como poco común y escasa. Es el caso también de Pandora bushiana que en las costas mexicanas está presente en Tamaulipas, Veracruz y Campeche, pero no en un estado intermedio entre los primeros y el último como lo es Tabasco. Lo mismo sucede con Cardiomya perrostrata y $C$. ornatissima (Pérez-Rodríguez 1997). No debe descartarse 
Tabla 1. Afinidad malacogeográfica de las especies de bivalvos marinos de la costa de Tamaulipas, México ( $N=131$ ) / Malacogeographic affinity of the marine bivalves species in Tamaulipas, Mexico $(\mathrm{N}=131)$

\begin{tabular}{lcc}
\hline Afinidad malacogeográfica & $\begin{array}{c}\text { Número de } \\
\text { especies }\end{array}$ & Porcentaje \\
\hline Principalmente Caribeña y alguna presencia Caroliniana & 62 & 47,32 \\
Caroliniana y Caribeña por igual & 20 & 15,26 \\
Principalmente Caribeña y alguna presencia Caroliniana y Argentiniana & 13 & 9,92 \\
Principalmente Caribeña y alguna presencia Caroliniana y Boreal & 12 & 9,16 \\
Boreal, Caroliniana y Caribeña & 11 & 8,40 \\
Exclusivamente Caribeña & 6 & 4,58 \\
Boreal, Caroliniana, Caribeña y Argentiniana & 4 & 3,05 \\
Ártica, Boreal, Caroliniana y Caribeña & 2 & 1,52 \\
Principalmente Caroliniana y alguna presencia Caribeña & 1 & 0,76 \\
\end{tabular}

que quizás sea necesario recolectar más en áreas específicas.

De las 4 especies $(3,05 \%$ del total) y de acuerdo al criterio de Leija-Tristán et al. (2000), distribución restringida al Golfo de México, se destacan 2 especies cuyos ámbitos de distribución geográfica son los más reducidos de todos (y además son continuos): Eurytellina alternata tayloriana en el área comprendida desde Texas (Abbott \& Morris 1995) a Quintana Roo (Isla Contoy) (Pérez-Rodríguez 1980); y Rangianella flexuosa desde Florida (Turgeon et al. 2009) hasta Campeche (Laguna de Términos), (Reguero 1994, Pérez-Rodríguez 1997).

La distribución geográfica de algunas especies se ha generalizado en la literatura. Por ejemplo Geukensia granosissima se ha registrado de Florida a Tamaulipas, y luego a Yucatán (Odé 1979, Andrews 1992, Abbott 1996, Mikkelsen \& Bieler 2004, García-Cubas \& Reguero 2007), pero no se ha señalado particularmente para el litoral de otro estado intermedio mexicano ni se ha indicado alguna localidad específica en esta zona. Aún más, para el caso de Tamaulipas y Yucatán, tampoco se ha mencionado en alguna localidad en particular (Abbott 1996, García-Cubas $\&$ Reguero 2007). Por tanto, en el presente trabajo se registra por primera vez en localidades específicas del estado de Tamaulipas, las cuales fueron Barra de Catán y La Pesca.

Con respecto a Crassostrea rhizophorae se le ha mencionado en el Golfo de México, particularmente para el manglar de la Isla del Carmen, Campeche (García-Cubas 1981), para las Lagunas de la Mancha y Alvarado (ambas en Veracruz) y Tupilco (Tabasco) según Reguero (1994) y Tamaulipas (García-Cubas \& Reguero 2007), sin precisar localidades. En este estudio se registran 4 localidades específicas, todas de Tamaulipas (Barra de Catán, La Pesca, Tepehuajes y Barra de Ostiones), lo que además de constituir nuevos registros de localidades en el Estado, señala el límite norteño (Barra de Catán, en el municipio de San Fernando) de su ámbito de distribución geográfica conocida, cuyo límite sureño es Uruguay (Abbott 1974).

En relación a Raetia plicatella se le conoce en la costa norteamericana del Golfo de México y de Tamaulipas a Yucatán (Odé 1975, Pérez-Rodríguez 1997, Rehder 2000, García-Cubas \& Reguero 2007), siendo la presencia en las localidades de La Pesca (municipio de Soto La Marina) y Barra del Tordo (municipio de Aldama), los primeros registros específicos conocidos para el estado de Tamaulipas.

En el caso de Rangianella flexuosa es conocida de Florida a Texas (Pulley 1952, Andrews 1977, Rehder 2000) y de Veracruz a Campeche (Odé 1975, García-Cubas 1981, García-Cubas \& Reguero 2007, Tunnel et al. 2010). Playa Bagdad, en el municipio de Matamoros, fue la primera localidad en particular conocida para Tamaulipas.

Por otra parte, aunque Andrews $(1977,1992)$ y Turgeon et al. (2009) mencionaron a Anatina anatina para México (en el último trabajo se le menciona para Veracruz), no se indica alguna localidad específica, por lo que su presencia en la Barra del Catán en el municipio de San Fernando, es la primera localidad georreferida para alguno de los estados mexicanos que colindan con el Golfo de México. 
En cuanto a Macrocallista nimbosa hallada para este estudio en Barra del Catán, San Fernando, constituye también, junto a Anatina anatina, un primer registro para Tamaulipas. En los estados mexicanos del Golfo de México se le conocía anteriormente, de acuerdo con PérezRodríguez (1997), desde el sur de la costa de Veracruz hasta la zona de Champotón, Campeche.

Sólo 3 especies (Eurytellina alternata tayloriana, Polymesoda maritima y Timoclea grus se distribuyen hasta el límite sureño de la provincia Mexicana que es reconocida por Pulley (1952), indicando que esta provincia se presenta desde Cabo Rojo (Isla de Enmedio, Veracruz) hasta Cabo Catoche (Quintana Roo). Once especies más, entre las que se hallan Anadara transversa, Tellidora cristata, Donax variabilis, Noetia ponderosa y Macrocallista nimbosa, se ubican en áreas cercanas a esta zona sur de la provincia Mexicana, al tener los límites sureños de sus ámbitos geográficos conocidos en estados cercanos, ya sea al oeste (Yucatán) o al suroeste (Campeche). Sin embargo, todas estas especies se ubican también hacia el norte, ya sea en Texas, Florida, Nueva York, Massachusetts e incluso el Ártico, mucho más allá del límite norte (Cabo Rojo) de la provincia Mexicana señalada por Pulley.

Lo anterior y el hecho de que las discontinuidades o rupturas de distribución en las costas mexicanas de las especies de este estudio sean excepcionales (e.g., Tellidora cristata, Polymesoda maritima y Geukensia granossisima) no permiten reconocer o confirmar con este trabajo la provincia malacológica Mexicana. Debe indicarse además, que no se presentan especies representativas o endemismos que la justifiquen como tal.

Por otro lado, las especies cuyos ámbitos de distribución geográfica se extienden a la provincia Ártica son sólo 2 especies (1,52\% del total) siendo éstas Yoldia sapotilla e Hiatella artica.

Cerca de una quinta parte de las especies (29: 22,39\%) extienden su distribución geográfica al norte de la provincia Caroliniana, es decir, a la provincia Boreal, específicamente a las subprovincias Virginiana o Acadiana (ésta última con presencia más al norte). La mayoría se encuentra en el primer caso, entre ellas Anadana transversa e Ischadium recurvum. En la subprovincia malacológica Acadiana se hallan Nucula proxima y Crassostrea virginica. En el sentido latitudinal opuesto, también son relativamente escasas las especies (17: 13\%) que presentan en su afinidad malacogeográfica algún componente correspondiente a la provincia Argentiniana.
Entre estos bivalvos se hallan Atrina seminuda y Dallocardia muricata.

Destacan 4 especies $(3,05 \%)$ de bivalvos marinos de las costas de Tamaulipas cuyos ámbitos de distribución geográfica conocidos son los más extensos en el Atlántico americano (provincias Boreal, Caroliniana, Caribeña y Argentiniana): Lunarca ovalis, Brachidontes recurvus, Crassostrea virginica y Petricolaria pholadiformis.

Por último, puede señalarse que los diversos métodos de muestreo empleados se complementaron adecuadamente y han permitido una revisión exhaustiva de esta malacofauna en el área de estudio.

\section{Agradecimientos}

A la Dirección General de Educación Superior Tecnológica por el financiamiento del proyecto. A Martha Reguero y Antonio García-Cubas del Laboratorio de Malacología del Instituto de Ciencias del Mar y Limnología, Universidad Nacional Autónoma de México y Adriana Reza del Texas State Aquarium por la literatura proporcionada. A Carmen Salazar, Gustavo Sarmiento, Rubén Rodríguez, Héctor Cepeda, Myrna Córdoba, Angelina Compean y Eros Salas por su apoyo en campo. A Ana Hernández por la elaboración de la figura. A dos revisores anónimos y a los editores cuyas observaciones, sugerencias y correcciones mejoraron sustancialmente el manuscrito.

\section{LITERATURA CITADA}

Abbott RT. 1970. How to know the American marine shells, 222 pp. The New American Library, New York.

Abbott RT. 1974. American seashells. The marine mollusks of the Atlantic and Pacific Coast of North America, 663 pp. Van Nostrand Reinhold Company, New York.

Abbott RT. 1996. Seashells of North America, 280 pp. St. Martin's Press, New York.

Abbott RT \& SP Dance. 2000. Compendium of seashells, 411 pp. Odyssey Publishing, El Cajón.

Abbott RT \& PA Morris. 1995. A field guide to shells of the Atlantic and Gulf Coasts and the West Indies, $350 \mathrm{pp}$. Houghton Mifflin Company, Boston.

Abbott RT \& H Zim. 1978. Conchas marinas, 160 pp. Ediciones Daimon, Manuel Tamayo, Barcelona.

Andrews J. 1977. Shells and shores of Texas, 365 pp. University of Texas Press, Austin.

Andrews J. 1992. A field guide to shells of the Texas Coast, 176 pp. Gulf Publishing Company, Houston. 
Britton JC \& B Morton. 1989. Shore ecology of the Gulf of Mexico, 387 pp. University of Texas Press, Austin.

Coan EV \& P Valentich-Scott. 2012. Bivalve seashells of tropical west America. Marine bivalve mollusks from Baja California to Peru. Monographs 6: 1-1258. Museum of Natural History, Santa Barbara.

De La Lanza-Espino G \& JC Gómez-Rojas. 2004. Características físicas y químicas del Golfo de México. En: Caso M, I Pisanty \& E Ezcurra (comp). Diagnóstico ambiental del Golfo de México, pp. 103-132. Secretaria del Medio Ambiente y Recursos Naturales, Instituto Nacional de Ecología, Instituto de Ecología, A. C., Harte Research Institute for Gulf of Mexico Studies, México.

Eckdale AA. 1974. Marine mollusks from the shallow-water environments $(0-60 \mathrm{~m})$ off the north east Yucatan Coast, Mexico. Bulletin of Marine Science 24(3): 638-668.

García-Cubas A. 1981. Moluscos de un Sistema Lagunar Tropical en el Sur del Golfo de México (Laguna de Términos, Campeche). Instituto de Ciencias del Mar y Limnología, Universidad Nacional Autónoma de México, Publicaciones Especiales 5: 1-182.

García-Cubas A \& M Reguero. 2007. Catálogo ilustrado de moluscos bivalvos del Golfo de México y Mar Caribe, 94 pp. Instituto de Ciencias del Mar y Limnología, Universidad Nacional Autónoma de México, México.

García-Cubas A, M Reguero \& L Jácome. 1994. Moluscos arrecifales de Veracruz, México (Guía de Campo), 143 pp. Instituto de Ciencias del Mar y Limnología, Universidad Nacional Autónoma de México, México.

García-Cubas A, F Escobar \& M Reguero. 1999. Gastrópodos marinos de la Península de Yucatán, México. Revista de la Sociedad Mexicana Historia Natural 49: 127-146.

Leal L. 1978. Estudio taxonómico de los moluscos (Gastropoda-Pelecypoda) representativos de la Región de Soto La Marina, Tamaulipas, México. Tesis de Licenciatura en Biología, Facultad de Ciencias Biológicas, Universidad Autónoma de Nuevo León, San Nicolás de los Garza, 107 pp.

Leija-Tristán A, A Contreras Arquieta, ME García-Garza, AJ Contreras-Balderas, MI Lozano-Vilano, S Contreras-Balderas, ME García-Ramírez, J OrtizRosales, F Segovia-Salinas, F Jiménez-Guzmán, D Lazcano-Villarreal, JA De León-González, S MartínezLozano, GA Rodríguez-Almaraz, MA Guzmán, MC González De La Rosa, JA García-Salas, G GuajardoMartínez, JI González-Rojas \& A Guzmán-Velazco. 2000. Taxonomic, bioecological and biogeographic aspects of selected biota of the Laguna Madre, Tamaulipas, Mexico. In: Munawar M, SG. Lawrence, IF Munawar \& DF Malley (eds). Aquatic ecosistems of Mexico, pp. 399-435. Ecovision World Monograph Series, Blackhuys Publishers, Leiden.
Llorente J \& JJ Morrone. 2001. Prefacio. En: Llorente J \& JJ Morrone (eds). Introducción a la biogeografía en Latinoamérica: Teorías, conceptos, métodos y aplicaciones, pp. i-ii. Comisión Nacional para el Conocimiento y Uso de la Biodiversidad, Universidad Nacional Autónoma de México, México.

Lomolino MV, BR Riddle, RJ Whittaker \& JH Brown. 2010. Biogeography, 878 pp. Sinauer Associates Incorporated, Sunderland.

Mikkelsen PM \& R Bieler. 2004. Critical catalog and annotated bibliography of marine bivalve records for the Florida Keys. Malacologia 46(2): 545-623.

Mikkelsen PM \& R Bieler. 2008. Seashells of Southern Florida. Living marine mollusks of the Florida Keys and adjacent regions. Bivalves, 503 pp. Princeton University Press, Princeton.

Morris PA. 1975. A field guide to shells of the Atlantic and Gulf Coast and the West Indies, 330 pp. Houghton Mifflin Company, Boston.

Odé H. 1975. Distribution and records of the marine Mollusca in the northwest Gulf of Mexico (a continuing monograph). Part I: Crassatellacea-Poromyarea. Texas Conchologist 12: $10-26$.

Odé H. 1979. Distribution and records of the marine Mollusca in the northwest Gulf of Mexico (a continuing monograph). Texas Conchologist 15: 45-56.

Pérez-Rodríguez R. 1980. Moluscos de la Plataforma Continental del Golfo de México y Caribe Mexicano. Tesis Doctoral, Facultad de Ciencias, Universidad Nacional Autónoma de México, México, 340 pp.

Pérez-Rodríguez R. 1997. Moluscos de la Plataforma Continental del Atlántico Mexicano. Universidad Autónoma Metropolitana, Unidad Xochimilco. Serie Académicos CBS 24: 1-260.

Petuch EJ. 2013. Biogeography and biodiversity of Western Atlantic mollusks, 252 pp. CRC Press, Boca Raton.

Pulley TE. 1952. An illustrated check list of the marine mollusks of Texas. The Texas Journal of Science 4(2): 167-199.

Redfern C. 2001. Bahamian seashells. A thousand species from Abaco, Bahamas, 280 pp. Bahamianseashells.com, Boca Ratón.

Reguero M. 1994. Estructura de la comunidad de moluscos en Lagunas Costeras de Veracruz y Tabasco, México. Tesis Doctoral, Facultad de Ciencias, Universidad Nacional Autónoma de México, México, 280 pp.

Rehder HA. 2000. Field guide to North American seashells, 849 pp. National Audubon Society, Chantticlee Press, Nueva York.

Rice WH. 1965. Mollusks from the deeper waters of the Northwestern Campeche Bank, Mexico. Publications of the Institute of Marine Sciences, University of Texas 10: 108-172. 
Rice WH \& L Kornicker. 1962. Mollusks of Alacran Reef, Campeche Bank, Mexico. Publications of the Institute of Marine Sciences, University of Texas 8: 388-403.

Sabelli B. 1980. Guide to shells, 512 pp. Simmon and Schuster, New York.

Shelton DN. 1997. Northern. A systematic list of mollusks in the Gulf of Mexico off the Coast of Alabama. Alabama Malacological Research Center, Alabama < http:// hiwaay.net/ dwills/marine/alamarsp.html>

Thorpe J. 2006. Editorial. Zoological Journal of the Linnean Society 148: 221.

Tunnell JW Jr \& AH Chaney. 1970. A checklist of mollusks of seven and one-half Fathom Reef, Northwest Gulf of Mexico. Contribution in Marine Science 15: 193-203.

Tunnell JW, J Andrews, NC Barrera \& F Moretzsohn. 2010. Encyclopedia of Texas seashells: Identification, ecology, distribution \& history, 512 pp. Texas A \& M University Press, College Station.
Turgeon DD, WG Lyons, P Mikkelsen, G Rosenberg \& F Moretzsohn. 2009. Bivalvia (Mollusca) of the Gulf of Mexico. In: Felder DL \& DK Kamp (eds). Gulf of Mexico. Origin, waters and biota. Vol. 1. Biodiversity, pp. 711-744. Texas A \& M University Press, College Station.

Vokes HE \& EH Vokes. 1983. Distribution of shallow-water marine mollusca, Yucatan, Peninsula, Mexico. Middle American Research Institute, Publication 54: 1-183.

Warmke GL \& RT Abbott. 1961. Caribbean seashells. A guide to the marine mollusks of Puerto Rico and other west Indian Islands, Bermuda and the Lower Florida Keys, 348 pp. Dover Publications, New York.

Recibido el 13 de enero 2013 y aceptado el 10 de octubre de 2013

Editor: Claudia Bustos D.

Apéndice 1. Coordenadas geográficas de las localidades muestreadas en la costa de Tamaulipas (Ver Fig. 1) / Geographical coordinates of the localities sampled in the coast of Tamaulipas (See Fig. 1)

\begin{tabular}{lll}
\hline Localidades & \multicolumn{2}{c}{ Coordenadas geográficas } \\
\hline 1. Playa Bagdad, Matamoros & $25^{\circ} 49^{\prime} 30^{\prime \prime} \mathrm{N}$ & $97^{\circ} 09^{\prime} 06^{\prime \prime} \mathrm{O}$ \\
2. Barra El Mezquital, Matamoros & $25^{\circ} 15^{\prime} 00^{\prime \prime} \mathrm{N}$ & $97^{\circ} 25^{\prime} 14^{\prime \prime} \mathrm{O}$ \\
3. Barra Los Americanos, San Fernando & $24^{\circ} 52^{\prime} 06^{\prime \prime} \mathrm{N}$ & $97^{\circ} 34^{\prime} 39^{\prime \prime} \mathrm{O}$ \\
4. Barra de Catán, San Fernando & $24^{\circ} 19^{\prime} 17^{\prime \prime} \mathrm{N}$ & $97^{\circ} 42^{\prime} 21^{\prime \prime} \mathrm{O}$ \\
5. Barra Soto La Marina, Soto La Marina & $24^{\circ} 03^{\prime} 00^{\prime \prime} \mathrm{N}$ & $97^{\circ} 43^{\prime} 50^{\prime \prime} \mathrm{O}$ \\
6. La Pesca, Soto La Marina & $23^{\circ} 42^{\prime} 06^{\prime \prime} \mathrm{N}$ & $97^{\circ} 44^{\prime} 23^{\prime \prime} \mathrm{O}$ \\
7. Tepehuajes, Soto La Marina & $23^{\circ} 30^{\prime} 00^{\prime \prime} \mathrm{N}$ & $97^{\circ} 45^{\prime} 31^{\prime \prime} \mathrm{O}$ \\
8. Barra de Ostiones, Soto La Marina & $23^{\circ} 25^{\prime} 14^{\prime \prime} \mathrm{N}$ & $97^{\circ} 45^{\prime} 56^{\prime \prime} \mathrm{O}$ \\
9. Barra del Tordo, Aldama & $23^{\circ} 03^{\prime} 13^{\prime \prime} \mathrm{N}$ & $97^{\circ} 45^{\prime} 42^{\prime \prime} \mathrm{O}$ \\
10. Barra de Morón, Aldama & $22^{\circ} 43^{\prime} 27^{\prime \prime} \mathrm{N}$ & $97^{\circ} 49^{\prime} 03^{\prime \prime} \mathrm{O}$ \\
11. Lomas del Real, Altamira & $22^{\circ} 31^{\prime} 15^{\prime \prime} \mathrm{N}$ & $97^{\circ} 51^{\prime} 29^{\prime}, \mathrm{O}$ \\
12. Playa de Altamira & $22^{\circ} 23^{\prime} 24^{\prime \prime} \mathrm{N}$ & $97^{\circ} 50^{\prime} 24^{\prime \prime} \mathrm{O}$ \\
13. Playa Miramar, Cd. Madero & $22^{\circ} 16^{\prime} 49^{\prime \prime} \mathrm{N}$ & $97^{\circ} 47^{\prime} 46^{\prime \prime} \mathrm{O}$ \\
\hline
\end{tabular}




\begin{abstract}
Apéndice 2. Afinidades malacogeográficas y distribución geográfica conocida de los bivalvos marinos de la costa de Tamaulipas. La afinidad exclusivamente Caribeña se simboliza 'Cñ', la Caroliniana y Caribeña 'CnCñ', la principalmente Caribeña y alguna presencia en la provincia Caroliniana 'CñpCn', la principalmente Caroliniana y alguna presencia Caribeña 'CnpCñ', la Boreal, Caroliniana y Caribeña 'BCnCñ', la principalmente Caribeña, Caroliniana y Boreal 'CñpCnB', la principalmente Caribeña, Caroliniana y Argentiniana 'CñpCnAg', la Ártica, Boreal, Caroliniana y Caribeña 'ABCnCñ', y la afinidad Boreal, Caroliniana, Caribeña y Argentiniana 'BCnCñAg' *Especie recolectada en este estudio. **Primer registro para Tamaulipas / Species of marine bivalves in Tamaulipas and their zoogeographical relationships. The Caribbean affinity is ' $C \tilde{n}$ '; both Carolinian and Caribbean affinities 'CnCñ'; Caribbean and Carolinian 'CñpCn'; Carolinian and Caribbean 'CnpCñ'; Boreal, Carolinian and Caribbean 'BCnCñ'; Caribbean, Carolinian and Boreal 'CñpCnB'; Caribbean, Carolinian and Argentinian 'CñpCnAg'; Artic, Boreal, Carolinian and Caribbean 'ABCnCñ'; and Boreal, Carolinian, Caribbean and Argentinian is 'BCnCñAg'. *Collected in this study. **First record for Tamaulipas
\end{abstract}

\section{FAMILIA NUCULIDAE}

Nucula proxima Say, 1822

Distribución Geográfica.- BCnCñ. De Nueva Escocia a Florida; Alabama; Texas; Tamaulipas (Playa Bagdad, Barra El Mezquital, Barra Los Americanos, Barra de Catán, Barra Soto La Marina, La Pesca, Barra de Ostiones, Barra del Tordo, Lomas del Real, Playa Miramar, Tampico); Veracruz (Pueblo Viejo, Cabo Rojo, Tuxpan); Tabasco (El Bosque, Puerto Real, Champotón); Bermudas.

\section{FAMILIA NUCULANIDAE}

Nuculana acuta (Conrad, 1832)

Distribución Geográfica.- CñpCnB. Cabo Cod, Massachussets; Alabama; Texas; Tamaulipas (Matamoros, San Fernando, La Pesca, Barra del Tordo, Barra de Morón, Playa de Altamira, Tampico); Veracruz (Laguna de Tamiahua; Pueblo Viejo, Cabo Rojo, Tuxpan, Laguna de Sontecomapan y hasta Coatzacoalcos); Tabasco (Complejo lagunar Carmen-Machona-Redonda); Campeche (Cd. del Carmen, El Bosque, Puerto Real, Laguna de Términos, Champotón); Quintana Roo; Caribe; Brasil.

\section{Nuculana concentrica (Say, 1824)}

Distribución Geográfica.- CñpCn. Noroeste de Florida; Alabama; Texas; Tamaulipas (Barra El Mezquital, Barra Los Americanos, Barra de Catán, Barra Soto La Marina, La Pesca, Lomas del Real, Playa Miramar, Tampico); Veracruz (toda la costa); Tabasco (Chiltepec); Campeche (El Burque, Puerto Real, Champotón, Campeche); Yucatán; Quintana Roo; Caribe hasta Brasil.

\section{FAMILIA YOLDIIDAE}

\section{Yoldia sapotilla (Gould, 1841)}

Distribución Geográfica.- ABCnCñ. De los mares del Ártico a Carolina del Norte; Tamaulipas (Playa Miramar, Tampico); Veracruz (zona norte hasta Tuxpan); Campeche (Champotón, Campeche).

\section{FAMILIA ARCIDAE}

\section{Acar dominguensis (Lamarck, 1819)}

Distribución Geográfica.- CñpCn. Carolina del Norte; Florida; Alabama; Texas; Tamaulipas (Matamoros a Playa Miramar, Tampico); Veracruz (toda la costa); Tabasco (Complejo lagunar Carmen-Machona-Redonda); Campeche (Sonda de Campeche, Champotón, Campeche, arrecifes Arcas, Arenas); Yucatán (Arrecife Alacranes, Celestún); Quintana Roo (Isla Mujeres); Costa Rica; Bermudas, Caribe; Surinam; Brasil y sus islas oceánicas.

Anadara baughmani (Hertlein, 1951)

Distribución Geográfica.- CñpCn. Alabama; Mississippi; Louisiana; Texas; Tamaulipas (Playa Bagdad, Barra El Mezquital, Barra Los Americanos, Barra Soto La Marina, La Pesca, Barra de Ostiones, Barra del Tordo, Lomas del Real, Playa Miramar, Tampico); Veracruz (Pueblo Viejo, Tuxpan); Campeche (Puerto Real, Champotón); Yucatán (Celestún, Sisal, Chelem, Yukalpeten, Chicxulub, Telchac, Dzilam, Río Lagartos); Quintana Roo (Holbox); Brasil.

Anadara floridana* (Conrad, 1869)

Distribución Geográfica.- CñpCn. Carolina del Norte; Florida a Texas; Tamaulipas (La Pesca, Tepehuajes, Barra del Tordo, Playa de Altamira, Playa Miramar); Veracruz (Playa El Pulpo, Barra de Cazones, Bajo de Hornos.; Lado sur de la desembocadura del Arroyo 'Río Grande'; Laguna de Sontecomapan y el resto del sur del estado); Tabasco (Laguna del Carmen, Chiltepec); Campeche (Laguna de Términos, El Bosque, Puerto Real, Champotón, Isla Arenas); Caribe.

Anadara notabilis* (Röding, 1798)

Distribución Geográfica.- CñpCn. Carolina del Norte a Florida; Alabama; Tamaulipas (Tepehuajes, Tampico); Veracruz (toda la costa); Campeche (El Bosque, Champotón, Seybaplaya, Sonda de Campeche, arrecife Arcas); Yucatán (Arrecife Alacranes, Celestún, Sisal, Chuburna, Chelem, Yukalpetén, Progreso, Chicxulub, Telchac, Dzilam, Río Lagartos); Quintana Roo (Holbox, Playa Carmen, Chetumal,); Bermudas; Brasil. 
Anadara transversa* (Say, 1822)

Distribución Geográfica.- BCnCñ. Del sur de Cabo Cod a Florida; Alabama; Texas; Tamaulipas (La Pesca, Tepehuajes, Barra de Morón, Playa de Altamira, Playa Miramar); Veracruz (Laguna de Pueblo Viejo; Laguna de Tamiahua, Laguna de Tampamachoco, Complejo lagunar Chica-Grande, Laguna de la Mancha, Laguna Camaronera, Laguna de Sontecomapan); Tabasco (Complejo lagunar Carmen-Machona-Redonda, Laguna de Mecoacán); Campeche (El Bosque, Sonda de Campeche, Laguna de Términos, Puerto Real, Champotón, Seybaplaya); Yucatán (Chelem y Chicxulub).

Arca imbricata* Bruguière, 1789

Distribución Geográfica.- CñpCn. Carolina del Norte; Florida; Alabama; Texas; Tamaulipas (La Pesca, Tepehuajes, Barra del Tordo, Barra de Morón, Playa de Altamira, Playa Miramar, Tampico); Veracruz (Isla Lobos, Tamiahua, Playa El Pulpo, Barra de Cazones, Bajo de Hornos; Sistema Arrecifal del Puerto de Veracruz); Campeche (Arrecifes Arcas, Triángulos, Arenas, Banco de Campeche, Laguna de Términos, Champotón, Cd. Sol, Seybaplaya); Yucatán (Arrecife Alacranes, Celestún, Sisal, Chuburna, Chelem, Yukalpetén, Progreso, Chicxulub, Telchac, Chavihau, Dzilam, Río Lagartos); Quintana Roo (Holbox, Isla Mujeres, Isla Contoy, Xcalac, Cancún, XelHa, Tulum, arrecife Chinchorro); Costa Rica; Bermudas; Caribe; Surinam; Brasil.

Arca zebra* (Swainson, 1833)

Distribución Geográfica.- CñpCn. Carolina del Norte; Florida; Alabama; Texas; Tamaulipas (La Pesca, Tepehuajes, Barra del Tordo, Barra de Morón, Playa de Altamira; Playa Miramar, Tampico); Veracruz (Playa El Pulpo, Barra de Cazones, Bajo de Hornos; Sistema Arrecifal del Puerto de Veracruz); Campeche (Arrecifes Arcas, Triángulos, Arenas, Banco de Campeche, Laguna de Términos, Champotón, Cd. Sol, Seybaplaya); Yucatán (Arrecife Alacranes Celestún, Sisal, Chuburna, Chelem, Yukalpetén, Progreso, Chicxulub, Telchac, Chavihau, Dzilam, Río Lagartos); Quintana Roo (Holbox, Isla Mujeres, Xcalac, arrecife Chinchorro); Costa Rica; Bermudas; Caribe; Surinam; Brasil; Bermudas.

Barbatia candida (Helbling, 1779)

Distribución Geográfica.- CñpCn. Carolina del Norte; Alabama; Texas; Tamaulipas (Playa de Altamira); Veracruz (Playa Paraíso; Sistema Arrecifal del Puerto de Veracruz); Tabasco (Complejo lagunar Tupilco-Ostión); Campeche (Arrecife Arcas); Caribe; Brasil.
Fugleria tenera (C.B. Adams, 1845)

Distribución Geográfica.- CñpCn. Sur de Florida a Texas; Tamaulipas (Playa de Altamira, Playa Miramar); Veracruz (zona norte y hasta Veracruz); Campeche (Champotón); Yucatán (Celestún), Caribe; Sudamérica.

Lunarca ovalis (Bruguiére, 1789)

Distribución Geográfica.- BCnCñAg. De Cabo Cod a Florida; Alabama; Texas; Tamaulipas (Tepehuajes, Tampico); Veracruz (Norte de Veracruz, Laguna de Sontecomapan); Tabasco (Complejo lagunar CarmenMachona-Redonda, Complejo lagunar Tupilco-Ostión, Laguna de Mecoacán); Campeche (El Bosque, Champotón, Campeche, Banco de Campeche); Yucatán; Quintana Roo; Caribe; Costa Rica; de la costa de Brasil a Rocha, Uruguay.

Scapharca brasiliana* (Lamarck, 1819)

Distribución Geográfica.- CñpCn. De Carolina del Norte al Oeste de Florida; Alabama; Texas; Tamaulipas (Playa Bagdad, Punta de Piedra, Barra de Catán, La Pesca, Tepehuajes, Barra de Ostiones, Barra del Tordo, Barra de Morón, Playa de Altamira, Miramar, Tampico); Veracruz (norte, centro y sur del estado); Tabasco (Complejo lagunar Carmen-Machona-Redonda; Laguna de Mecoacán, Chiltepec); Campeche (El Bosque, Puerto Real, Seybaplaya, Campeche); Yucatán (Celestún); Quintana Roo; Caribe; Surinam; Brasil.

Scapharca chemnitzii* (Philippi, 1851)

Distribución Geográfica.- CñpCn. Sur de Florida; Texas; Tamaulipas (Barra de Catán, La Pesca, Tepehuajes, Barra del Tordo); Veracruz (Playa Bonita, Tuxpan; Complejo lagunar Chica-Grande, Playa El Pulpo, Barra de Cazones, Bajo de Hornos); Tabasco (Complejo lagunar CarmenMachona-Redonda); Campeche (Champotón, Campeche); Caribe; Surinam; Brasil.

\section{FAMILIA NOETIIDAE}

Arcopsis adamsi (Dall, 1886)

Distribución Geográfica.- CñpCn. Carolina del Norte al oeste de Florida, Alabama, Texas; Tamaulipas (Barra El Mezquital, Barra Soto La Marina, La Pesca, Barra de Ostiones, Lomas del Real, Playa Miramar, Tampico); Veracruz (Toda la costa); Tabasco (Complejo lagunar Carmen-Machona-Redonda); Campeche (arrecife Arenas, Champotón, Seybaplaya); Yucatán (Celestún; Quintana Roo (Isla Mujeres); Caribe; Brasil. 
Noetia ponderosa* (Say, 1822)

Distribución Geográfica.- CnpCñ. De Virginia a Cayos del Oeste, Florida; Alabama; Texas; Tamaulipas (La Pesca, Tepehuajes, Barra del Tordo, Barra de Morón, Playa de Altamira; Playa Miramar); Veracruz (Laguna de Pueblo Viejo); Tabasco (Complejo lagunar Carmen-MachonaRedonda); Campeche (Champotón, Cd. Sol, Seybaplaya, Campeche); Yucatán.

\section{FAMILIA GLYCYMERIDIDAE}

\section{Glycymeris americana (De France, 1829)}

Distribución Geográfica.- CñpCn. Carolina del Norte; Florida; Alabama; Texas; Tamaulipas (La Pesca, Barra de Morón, Playa de Altamira, Playa Miramar, Tampico); Veracruz (toda la costa); Tabasco (Laguna del Carmen, Laguna Machona, Chiltepec); Campeche (Laguna de Términos, El Bosque, Puerto Real, Champotón, Campeche); Yucatán (Celestún); Brasil.

Tucetona pectinata* (Gmelin, 1791)

Distribución Geográfica.- CñpCn. Desde Carolina del Norte; Alabama; Tamaulipas (Tepehuajes, Tampico); Veracruz (toda la costa); Tabasco (Complejo lagunar Carmen-Machona-Redonda, Chiltepec); Campeche (desde El Bosque a Campeche); Yucatán (Celestún); Caribe hasta Brasil.

\section{FAMILIA MYTILIDAE}

\section{Brachidontes exustus (Linnaeus, 1758)}

Distribución Geográfica.- CñpCnAg. Carolina del Norte; Alabama; Texas; Tamaulipas (Laguna Madre, La Pesca, Tepehuajes, Barra de Morón, Playa de Altamira, Playa Miramar, Tampico); Veracruz (Laguna de Pueblo Viejo; Laguna Tampamachoco; Complejo lagunar Chica-Grande; Playa Paraíso; Complejo lagunar Larga-Redonda o Mandinga Chica-Mandinga Grande; Laguna de Alvarado; Laguna Sontecomapan); Tabasco ( Complejo lagunar Carmen-Machona-Redonda; Complejo lagunar TupilcoOstión; Laguna de Mecoacán); Campeche (Laguna de Términos, El Bosque, Puerto Real); Yucatán; Quintana Roo; Caribe; de Brasil a Uruguay.

Brachidontes recurvus (Linnaeus, 1758)

Distribución Geográfica.- BCnCñAg. Cabo Cod; Texas; Tamaulipas (La Pesca, Barra de Ostiones, Tepehuajes, Barra del Tordo); Veracruz (Salado Chico, Playa El Pulpo, Barra de Cazones, Bajo de Hornos); Campeche (Laguna de Téminos); Yucatán; Caribe; de Brasil a Uruguay.
Geukensia granosissima* (Sowerby III, 1914)

Distribución Geográfica.- CnCñ. De ambos lados de Florida a Texas; Tamaulipas (Barra de Catán, La Pesca); Yucatán.

Ischadium recurvum* (Rafinesque, 1820)

Distribución Geográfica.- BCnCñ. Cabo Cod; Alabama; Texas; Tamaulipas (La Pesca, Barra del Tordo, Barra de Morón, Playa de Altamira, Playa Miramar, Tampico); Veracruz (Laguna de Pueblo Viejo, Laguna de Tamiahua, Laguna Tampamachoco, Tuxpan, Nautla, Complejo lagunar Chica-Grande, Laguna de la Mancha, Playa Paraíso, Complejo lagunar Larga-Redonda o Mandinga Chica-Mandinga Grande, Laguna Camaronera, Laguna de Alvarado, Coatzacoalcos); Tabasco (Complejo lagunar Carmen-Machona-Redonda; Complejo Lagunar TupilcoOstión; Laguna de Mecoacán); Campeche (El Bosque, Cd. del Carmen; Puerto Real, Laguna de Términos); Caribe.

Lithophaga aristata (Dillwyn, 1817)

Distribución Geográfica.- CnCñ. De Carolina del Norte a Florida; Alabama; Texas; Tamaulipas (La Pesca, Playa de Altamira, Playa Miramar); Veracruz (Isla Lobos, Sistema Arrecifal del Puerto de Veracruz); Campeche (Champotón); Yucatán (Celestún, Progreso); Quintana Roo (Isla Contoy, Isla Mujeres, Cancún, Isla Cozumel); Caribe.

Modiolus americanus* (Leach, 1815)

Distribución Geográfica.- CñpCn. Carolina del Norte; Florida; Texas; Tamaulipas (La Pesca, Barra del Tordo, Barra de Morón, Playa de Altamira, Playa Miramar); Veracruz (lado sur de la desembocadura del Arroyo 'Río Grande', Bajo de Hornos, Sistema Arrecifal del Puerto de Veracruz); Campeche (Arrecife Alacranes del Banco de Campeche y el propio Banco de Campeche, Laguna de Términos, Champotón, Campeche); Yucatán (Celestún, Telchac, Río Lagartos, Arrecife Alacranes); Quintana Roo (Holbox); Bermudas; Surinam; Brasil.

\section{FAMILIA PTERIIDAE}

Pinctada imbricata Röding, 1798

Distribución Geográfica.- CñpCn. Carolina del Sur a Florida; Texas; Tamaulipas (Playa de Altamira); Veracruz (zona norte de Veracruz, Playa Paraíso); Campeche (El Bosque, Laguna de Términos, Champotón); Yucatán (Chelem, Yukalpetén, Chicxulub, Telchac, Dzilam, Río Lagartos); Quintana Roo (Holbox, Playa Carmen); Bermudas; Caribe; Brasil. 
Pteria colymbus (Röding, 1798)

Distribución Geográfica.- CñpCn. De Carolina del Norte a Florida; Alabama; Texas; Tamaulipas (Barra de Morón, Barra del Tordo, Playa de Altamira, Playa Miramar, Tampico); Veracruz (Cabo Rojo a Arrecife Blanquita); Campeche (El Bosque, Puerto Real, Champotón); Yucatán (Celestún, Progreso, Chicxulub, Telchac, Dzilam, Río Lagartos); Quintana Roo (Holbox); Costa Rica; Bermudas; Caribe; Brasil.

\section{FAMILIA ISOGNOMONIDAE}

Isognomon alatus (Gmelin, 1791)

Distribución Geográfica.- CñpCn. Porción centro suroeste de Florida; Alabama; Texas; Tamaulipas (Playa de Altamira); Veracruz (toda la costa); Tabasco (Complejo lagunar Carmen-Machona-Redonda, Complejo lagunar Tupilco-Ostión, Laguna de Mecoacán, Chiltepec); Campeche (Banco de Campeche, Laguna de Términos, Champotón, arrecife Arcas); Yucatán (Celestún, Arrecife Alacranes, Dzilam); Quintana Roo (Xcaret, Cozumel); Centroamérica; Bermudas; Caribe; Surinam; islas oceánicas de Brasil.

Isognomon bicolor (C. B. Adams, 1845)

Distribución Geográfica.- CñpCn. Florida; Texas; Tamaulipas (Playa Miramar); Veracruz (Playa Paraíso; Laguna de Sontecomapan, Veracruz); Campeche (Banco de Campeche, Champotón, arrecifes Arcas, Triángulos, Arenas); Yucatán (Celestún, Chelem, Progreso, Dzilam, Arrecife Alacranes); Quintana Roo (Contoy); Costa Rica; Bermudas; Caribe.

Perna perna (Linnaeus, 1758)

Distribución Geográfica.- CñpCn. Texas, Tamaulipas (Laguna Madre, La Pesca, Barra de Ostiones, Barra del Tordo); Veracruz; área sur del Caribe; Sudamérica.

\section{FAMILIA OSTREIDAE}

Crassostrea rhizophoraea* (Guilding, 1828)

Distribución Geográfica.- CñpCnAg. Texas; Tamaulipas (Barra de Catán, La Pesca, Barra de Ostiones, Tepehuajes); Veracruz (Lagunas La Mancha y Alvarado); Tabasco (Laguna Tupilco); Campeche (Laguna de Términos); Quintana Roo; del Caribe a Brasil y Uruguay.

Crassostrea virginica* (Gmlein, 1791)

Distribución Geográfica.- BCnCñAg. Canadá (Golfo de San Lorenzo); Alabama; Texas; Tamaulipas (Laguna Madre, La Pesca, Laguna de Morales, Barra de Ostiones,
Tepehuajes, Barra del Tordo, Barra de Morón, Playa de Altamira, Playa Miramar, Tampico); Veracruz (toda la costa); Tabasco (Complejo lagunar Carmen-MachonaRedonda; Complejo lagunar Tupilco-Ostión; Laguna de Mecoacán); Campeche (Puerto Real, Laguna de Términos); Caribe; Brasil; Argentina.

Ostrea equestris* Say, 1834

Distribución Geográfica.- CñpCnAg. Alabama; Texas; Tamaulipas (Tepehuajes, Barra del Tordo, Barra de Morón); Tabasco (Complejo lagunar Carmen-MachonaRedonda); Campeche (Laguna de Términos); Caribe; de Brasil a Argentina.

\section{FAMILIA PINNIDAE}

Atrina rigida* (Lightfoot, 1786)

Distribución Geográfica.- CnCñ. De Carolina del Norte al Suroeste de Florida; Alabama; Texas; Tamaulipas (La Pesca, Tepehuajes, Barra de Morón, Tampico); Veracruz (Pueblo Viejo, Nautla, Bajo de Hornos, El Playón, Sistema Arrecifal del Puerto de Veracruz); Tabasco (La Machona, Chiltepec); Campeche (Puerto Real, Laguna de Términos, Champotón); Yucatán (Celestún, Sisal, Progreso, Chicxulub, Telchac, Dzilam, Río Lagartos); Quintana Roo (Holbox); Caribe.

Atrina seminuda (Lamarck, 1819)

Distribución Geográfica.- CñpCnAg. Carolina del Norte; Alabama; Texas; Tamaulipas (La Pesca, Tepehuajes, Barra del Tordo, Playa de Altamira); Veracruz (Playa El Pulpo, Barra de Cazones, Bajo de Hornos, Laguna de Sontecomapan); Campeche (Laguna de Términos); Yucatán; Caribe; Surinam; de Brasil a Argentina.

Atrina serrata* (G. B. Sowerby I, 1825)

Distribución Geográfica.- CnCñ. De Carolina del Norte a Florida; Alabama; Texas; Tamaulipas (La Pesca, Tepehuajes, Barra del Tordo, Barra de Morón, Playa de Altamira, Tampico); Veracruz (de Pueblo Viejo a Tecolutla); Campeche (Laguna de Términos); Quintana Roo (Boca Iglesias); Caribe; Surinam.

\section{FAMILIA LIMIDAE}

Lima lima (Linnaeus, 1758)

Distribución Geográfica.- CñpCn. Carolina del Sur a Texas; Sureste de Florida; Tamaulipas (Playa Miramar, Tampico); Veracruz (Costa norte y centro); Campeche (Puerto Real, Champotón, Arrecife Arcas); Yucatán (Celestún); Quintana Roo (Playa Carmen, Isla Mujeres y Arrecife Chinchorro); Caribe; Brasil. 


\section{FAMILIA PECTINIDAE}

Aequipecten muscosus* (Wood, 1828)

Distribución Geográfica.- CñpCn. De Carolina del Norte a Florida; Alabama; Texas; Tamaulipas (La Pesca, Tepehuajes, Barra de Morón); Tabasco (Complejo lagunar Carmen-Machona-Redonda); Campeche (Banco de Campeche, Laguna de Términos); Yucatán (Celestún, Yukalpetén, Chicxulub, Telchac, Río Lagartos); Quintana Roo (Holbox); Bermudas; Caribe; Brasil.

Argopecten gibbus* (Linnaeus, 1758)

Distribución Geográfica.- CñpCn. De Carolina del Norte a Florida; Alabama; Texas; Tamaulipas (Carbonera, Punta de Piedra, Barra de Catán, La Pesca, Tepehuajes, Barra de Ostiones, Barra del Tordo, Barra de Morón, Playa Miramar); Veracruz (Tuxpan; Playa El Pulpo, Barra de Cazones, Bajo de Hornos); Tabasco (Complejo lagunar Carmen-Machona-Redonda); Campeche (Sonda de Campeche; Banco de Campeche); Yucatán (Chicxulub, Río Lagartos); Quintana Roo (Holbox); Bermudas; Caribe; Brasil.

Argopecten irradians amplicostatus* (Dall, 1898)

Distribución Geográfica.- CñpCn. Centro y sur de Texas; Tamaulipas (La Pesca, Tepehuajes, Barra del Tordo, Barra de Morón, Playa de Altamira; Playa Miramar); Veracruz (Tuxpan); Colombia.

Argopecten nucleus (Born, 1778)

Distribución Geográfica.- Cñ. Sureste de Florida; Tamaulipas (Playa Miramar, Tampico); Veracruz (toda la costa); Campeche (El Bosque, Puerto Real, Champotón, Campeche); Caribe.

Caribachlamys sentis (Reeve, 1835)

Distribución Geográfica.- CñpCn. Carolina del Norte al Sur de Florida; Tamaulipas (Tepehuajes,); Veracruz (Sistema Arrecifal del Puerto de Veracruz); Bermudas; Caribe; Brasil.

Euvola cf. papyracea (Gabb, 1873)

Distribución Geográfica.- CñpCn. Carolina del Norte; Alabama; Texas; Tamaulipas (Playa de Altamira, Tampico); Veracruz (toda la costa); Campeche (El Bosque, Puerto Real, Champotón, Campeche); Caribe; Brasil

Euvola raveneli (Dall, 1898)

Distribución Geográfica.- CnCñ. Carolina del Norte a Florida; Alabama; Texas; Tamaulipas (Tepehuajes, Barra de Morón, Playa de Altamira); Veracruz (costa sur); Tabasco (Laguna del Carmen); Campeche (El Bosque, Puerto Real, Champotón, Campeche, arrecife Arenas); Yucatán (Sisal, Telchac); Caribe.

Nodipecten fragosus* (Linnaeus, 1758)

Distribución Geográfica.- CñpCnB. Maryland a Florida; Texas; Tamaulipas (La Pesca, Tepehuajes, Barra del Tordo, Barra de Morón, Playa de Altamira, Playa Miramar, Tampico); Veracruz (Costa norte y centro); Campeche (Puerto Real, Champotón); Yucatán (Sisal, Telchac, Las Coloradas); Quintana Roo (Holbox); Bermudas, Caribe; Surinam; Isla Ascensión, parte media del Atlántico; Brasil.

FAMILIA SPONDYLIDAE

Spondylus americanus* (Hermann, 1781)

Distribución Geográfica.- CñpCn. De Carolina del Norte a Florida; Alabama; Texas; Tamaulipas (La Pesca, Tepehuajes, Barra del Tordo, Playa de Altamira, Playa Miramar); Veracruz (Lado sur de la desembocadura del Arroyo 'Río Grande'; zona entre la desembocadura del Río Medio y Vergara; Playa El Pulpo, Barra de Cazones, Bajo de Hornos; Sistema Arrecifal del Puerto de Veracruz, Isla Verde, Arrecife Blanquita); Campeche (Banco de Campeche, Champotón, arrecifes Arcas, Triángulos, Arenas); Yucatán (Celestún, Arrecife Alacranes); Quintana Roo (Playa Carmen); América Central; Caribe; Brasil.

\section{FAMILIA PLICATULIDAE}

Plicatula gibbosa Lamarck, 1801

Distribución Geográfica.- CñpCnAg. De Carolina del Norte a Florida; Alabama; Texas; Tamaulipas (Barra El Mezquital, Barra Soto La Marina, La Pesca, Barra de Ostiones, Barra del Tordo, Lomas del Real, Tampico, Altamira); Veracruz (costa norte y centro); Tabasco (Chiltepec); Campeche (Banco de Campeche, Laguna de Términos, Puerto Real, Champotón, El Sol, Seybaplaya, Campeche); Yucatán (Celestún, Sisal, Chuburna, Chelem, Yukalpetén, Progreso, Chicxulub, Telchac, Chavihau, Dzilam, Río Lagartos, arrecife Alacranes); Quintana Roo (Holbox, Isla Mujeres, Playa Carmen, arrecife Chinchorro); Bermudas, Caribe; Surinam; Brasil; Uruguay.

\section{FAMILIA ANOMIIDAE}

Anomia simplex* D’ Orbigny, 1853

Distribución Geográfica.- CñpCnB. De Cabo Cod, Massachussets a Florida; Alabama; Texas; Tamaulipas (Barra El Mezquital, La Pesca, Tepehuajes, Barra del 
Tordo, Playa de Altamira, Playa Miramar, Tampico); Veracruz (toda la costa); Tabasco (Complejo lagunar Carmen-Machona-Redonda, Chiltepec); Campeche (El Bosque, Sonda de Campeche; Banco de Campeche, Laguna de Términos, Champotón, Campeche); Yucatán (Celestún, Sisal, Chuburna, Chelem, Yukalpetén, Progreso, Chicxulub, Telchac, Chavihau, Dzilam, Río Lagartos, Arrecife Alacranes, Punta Arenas); Quintana Roo (Holbox, Isla Contoy, Mujeres, Cozumel, Cancún); Bermudas; Caribe; Surinam; Brasil.

Pododesmus rudis (Broderip, 1834)

Distribución Geográfica.- CñpCnAg. Carolina del Sur; Florida; Texas; Tamaulipas (Playa de Altamira); Bermudas; Brasil; Argentina.

\section{FAMILIA CRASSATELLIDAE}

Crassinella lunulata (Conrad, 1834)

Distribución Geográfica.- CñpCnB. Del Cabo Cod al Sur de Florida; Alabama; Texas; Tamaulipas (Playa Miramar, Tampico); Veracruz (toda la costa); Tabasco (Complejo lagunar Carmen-Machona-Redonda y Tupilco-Ostión, Chiltepec); Campeche (El Bosque, Puerto Real, Champotón, Laguna de Términos); Yucatán; Quintana Roo; Bermudas; Caribe; Brasil.

\section{FAMILIA CARDITIDAE}

Carditamera floridana* Conrad, 1838

Distribución Geográfica.- CñpCn. Sur de Florida; Alabama; Texas; Tamaulipas (Playa Bagdad, La Pesca, Barra de Ostiones, Tepehuajes, Barra del Tordo, Tampico); Veracruz (toda la costa); Campeche (El Bosque, Laguna de Términos, Cd. Sol, Seybaplaya, Champotón); Yucatán (Celestún, Sisal, Chuburna, Chelem, Yukalpeten, Progreso, Chicxulub, Telchac, Chavihau, Dzilam, Río Lagartos, Quintana Roo (Holbox, Contoy, Isla Mujeres, Cancún); Brasil.

Cardita gracilis (Schuttleworth, 1856)

Distribución Geográfica.- Cñ. Florida; Tamaulipas (La Pesca); Costa Rica; Caribe; Puerto Rico.

\section{FAMILIA PANDORIDAE}

\section{Pandora bushiana Dall, 1886}

Distribución Geográfica.- CñpCn. De Carolina del Norte a Florida; Texas; Tamaulipas (Barra El Mezquital, Barra Soto La Marina, La Pesca, Playa Miramar, Tampico); Veracruz (Pueblo Viejo a Punta Delgada, Coatzacoalcos); Campeche (Puerto Real, Champotón, Campeche); Caribe; Brasil.

\section{FAMILIA PERIPLOMATIDAE}

Periploma margaritaceum (Lamarck, 1801)

Distribución Geográfica.- CnpCñ. De Carolina del Sur a Florida; Texas; Tamaulipas (Barra El Mezquital); Costa Rica; Caribe; Brasil.

\section{FAMILIA VERTICORDIIDAE}

Trigonulina ornata (Orbigny, 1842)

Distribución Geográfica.- CñpCnB. De Massachussets a Florida; Tamaulipas (Playa Bagdad, Barra El Mezquital, Barra Los Americanos, Barra Soto La Marina, La Pesca, Barra de Ostiones, Barra del Tordo, Aldama, Lomas del Real, Tampico); Veracruz (de Pueblo Viejo a Punta Delgada, Alvarado a Coatzacoalcos); Tabasco (Chiltepec); Campeche (El Bosque, Punta Real, Champotón); Bermudas; Caribe; Brasil.

\section{FAMILIA CUSPIDARIIDAE}

Cardiomya ornatissima (D’Orbigny, 1842)

Distribución Geográfica.- CnCñ. Carolina de Norte; Texas; Tamaulipas (Playa Bagdad, Barra El Mezquital, Barra Los Americanos, Barra de Catán, Barra Soto La Marina; La Pesca, Barra de Ostiones, Barra del Tordo, y Lomas del Real, Tampico); Veracruz (Pueblo Viejo a Punta del Morro, Alvarado, Coatzacoalcos); Campeche (El Bosque, Puerto Real, Champotón, Campeche); Yucatán; Caribe.

Cardiomya perrostrata (Dall, 1881)

Distribución Geográfica.- BCnCñ. Massachusetts; Tamaulipas (Barra El Mezquital, Barra Los Americanos, Barra de Catán, Barra Soto La Marina, La Pesca, Barra de Ostiones, Barra del Tordo y Lomas del Real, Tampico); Veracruz (Pueblo Viejo a Punta Delgada, Alvarado, Coatzacoalcos), Campeche (El Bosque, Puerto Real, Champotón); Caribe.

\section{FAMILIA LUCINIDAE}

Anodontia alba* Link, 1807

Distribución Geográfica.- CñpCn. Carolina del Norte; Florida; Alabama; Texas; Tamaulipas (Punta de Piedra, Barra de Catán, La Pesca, Tepehuajes, Barra del Tordo, Playa Miramar, Tampico); Veracruz (toda la costa); Tabasco (Laguna del Carmen, Laguna Machona);Campeche (El Bosque, Sonda de Campeche y Laguna de Términos, Puerto Real, Champotón, Arrecife Arcas); Yucatán (Celestún, Sisal, Chubuma, Chelem, Yukalpeten, Progreso, Chicxulub, Telchac, Chavihau, Dzilam, Río Lagartos; Quintana Roo (Holbox); Costa Rica; Bermudas; Caribe; Brasil. 
Pegophysema philippiana* (Reevé, 1850)

Distribución Geográfica.- CnCñ. De Carolina del Norte a Florida; Texas; Tamaulipas (Punta de Piedra, La Pesca, Tepehuajes, Barra de Ostiones, Barra del Tordo, Barra de Morón, Playa de Altamira, Playa Miramar); Veracruz (Playa El Pulpo, Barra de Cazones, Bajo de Hornos, Veracruz; Campeche (Banco de Campeche, Laguna de Términos, Campeche); Yucatán; Costa Rica; Centroamérica; Bermudas, Cuba; Caribe.

Codakia orbicularis* (Linnaeus, 1758)

Distribución Geográfica.- CñpCn. De Carolina del Norte a Florida; Alabama; Texas; Tamaulipas (Punta de Piedra, La Pesca, Tepehuajes, Barra del Tordo, Playa de Altamira, Tampico); Veracruz (toda la costa); Campeche (El Bosque, Banco de Campeche, Sonda de Campeche, Puerto Real, Laguna de Términos, Champotón, Campeche, arrecifes Arcas, Triángulos, Arenas); Yucatán (Arrecife Alacranes, Celestún, Sisal, Chubuma, Chelem, Yukalpetén, Progreso, Chicxulub, Telchac, Chavihau, Dzilam); Quintana Roo (Holbox, Contoy, Isla Mujeres, Playa Carmen, Xcalac, Chetumal, Isacos, Majahual y arrecife Chinchorro); Costa Rica; Bermudas; Brasil.

Divaricella quadrisulcata (D’Orbigny, 1846)

Distribución Geográfica.- BCnCñ. De Massachussets al Sur de Florida; Alabama; Tamaulipas (Playa Miramar, Tampico); Veracruz (toda la costa); Campeche (Puerto Real, Champotón, Campeche, Arrecife Arenas); Yucatán (Celestún, Chicxulub, Telchac, arrecife Alacranes); Quintana Roo (Contoy, Playa Carmen); Caribe; Brasil.

Linga amiantus (Dall, 1901)

Distribución Geográfica.- CñpCn. De Carolina de Norte a ambos lados de Florida; Alabama; Texas; Tamaulipas (Playa Miramar, Tampico); Veracruz (costa norte); Campeche (El Bosque, Puerto Real, Champotón); Caribe; Brasil.

\section{Phacoides pectinata* (Gmelin, 1791)}

Distribución Geográfica.- CñpCn. Carolina del Norte a Florida; Alabama; Texas; Tamaulipas (La Pesca, Tepehuajes, Barra del Tordo, Barra de Morón, Playa de Altamira, Playa Miramar); Veracruz (Laguna de Tamiahua, Laguna Tampamachoco, Complejo lagunar Chica-Grande, Laguna de la Mancha, Laguna Camaronera, Laguna Sontecomapan); Tabasco (Complejo lagunar CarmenMachona-Redonda, Complejo lagunar Tupilco-Ostión, Laguna de Mecoacán); Campeche (Laguna de Términos, Champotón, Seybaplaya); Yucatán (Celestún, Chicxulub,
Dzilam); Quintana Roo (Holbox, Cancún); Caribe; Centroamérica a Brasil.

Parvilucina crenella (Dall, 1901)

Distribución Geográfica.- CñpCn. De Carolina del Norte a ambos lados de Florida; Alabama; Texas; Tamaulipas (Playa Miramar); Campeche (Laguna de Términos); Yucatán; Quintana Roo; Brasil.

\section{FAMILIA CHAMIDAE}

Arcinella arcinella (Linné, 1767)

Distribución Geográfica.- CnpCñ. Carolina del Norte; ambos lados de Florida; Texas; Tamaulipas (La Pesca).

Arcinella cornuta* Conrad, 1866

Distribución Geográfica.- CñpCn. De Carolina del Norte a ambos lados de Florida; Alabama; Texas; Tamaulipas (Barra El Mezquital, Punta de Piedra, Barra de Catán, La Pesca, Barra de Ostiones, Tepehuajes, Barra del Tordo, Barra de Morón, Playa de Altamira; Playa Miramar, Tampico); Veracruz (zona entre la desembocadura del Río Medio y Vergara; Tuxpan, Playa El Pulpo, Barra de Cazones, Bajo de Hornos, Arrecife Blanquita; Isla Verde); Campeche (Sonda de Campeche; Cd. Del Carmen; Laguna de Términos, Champotón); Yucatán (Celestún, Sisal y Progreso); Caribe; Brasil.

Chama radians (Lamarck, 1819)

Distribución Geográfica.- CnCñ. Carolina del Norte; Alabama; Texas; Tamaulipas (Tepehuajes, Playa Miramar); Campeche (arrecifes Arcas, Triángulos, Arenas); Yucatán (Celestún, Sisal, Chelem, Yukalpeten, Chicxulub, Arrecife Alacranes, Telchac); Quintana Roo (Holbox, Playa Carmen), Bermudas; Caribe.

Chama sinuosa Broderip, 1836

Distribución Geográfica.- Cñ. Sureste de Florida; Tamaulipas (Lomas del Real, Altamira, Tampico); Veracruz (Isla Lobos, Tecolutla, Veracruz, Arrecife Blanquita, Isla Verde); Yucatán (Celestún, Sisal, Chelem, Yukalpetén y Chicxulub); Bermudas; Caribe; Brasil.

\section{FAMILIA LASAEIDAE}

Aligena texasiana Harry, 1969

Distribución Geográfica.- CnCñ. Louisiana; Texas; Tamaulipas (Playa Miramar, Tampico); Veracruz (toda la costa), Campeche (El Bosque, Laguna de Términos, Champotón). 


\section{FAMILIA HIATELLIDAE}

Hiatella artica (Linnaeus, 1767)

Distribución Geográfica.- ABCnCñ. Desde los Mares del Polo Ártico hasta Caribe; Tamaulipas (Playa Miramar, Tampico); Veracruz (toda la costa); Campeche (Champotón); Yucatán; Brasil.

\section{FAMILIA CORBICULIDAE}

Polymesoda caroliniana (Bosc, 1801)

Distribución Geográfica.- CnpCñ. Virgina; norte de Florida; Alabama; Texas; Tamaulipas (Barra de Morón, Playa de Altamira); Veracruz (Laguna de Pueblo Viejo, Laguna de Alvarado); Tabasco (Complejo lagunar Carmen-MachonaRedonda); Campeche (Laguna de Términos).

Polymesoda maritima (D’Orbigny, 1853)

Distribución Geográfica.- CnCñ. Noreste de Florida; Texas; Tamaulipas (Playa de Altamira); Yucatán (Celestún, Yukalpeten, Telchac, Chavihau, Dzilam, Río Lagartos, Xcalac); Quintana Roo.

\section{FAMILIA CARDIIDAE}

Americardia media (Linnaeus, 1758)

Distribución Geográfica.- CñpCn. De Carolina del Norte al Sureste de Florida; Alabama; Tamaulipas (Tepehuajes); Veracruz (Sistema Arrecifal del Puerto de Veracruz); Bermudas; Caribe; Brasil.

Dallocardia muricata* (Linnaeus, 1758)

Distribución Geográfica.- CñpCnAg. De Carolina del Norte; Alabama; Texas; Tamaulipas (La Pesca, Tepehuajes, Barra del Tordo, Barra de Morón, Playa de Altamira, Playa Miramar, Tampico); Veracruz (toda la costa); Tabasco (Complejo lagunar Carmen-Machona-Redonda); Campeche (El Bosque, Laguna de Términos, Puerto Real, Champotón); Yucatán (Celestún, Sisal, Chelem, Yukalpetén, Progreso, Chicxulub, Río Lagartos); Quintana Roo (Playa Carmen); Caribe; Surinam; Brasil; Uruguay.

Dinocardium robustum* (Ligthfoot, 1786)

Distribución Geográfica.- BCnCñ. De Virginia al Norte de Florida; Alabama; Texas; Tamaulipas (Carbonera, Punta de Piedra, La Pesca, Barra de Ostiones, Tepehuajes, Barra del Tordo, Barra de Morón, Playa de Altamira, Playa Miramar, Tampico); Veracruz (Playa El Pulpo, Barra de Cazones, Bajo de Hornos y hasta Coatzacoalcos); Tabasco (Complejo lagunar Carmen-Machona-Redonda; Complejo lagunar Tupilco-Ostión; Laguna de Mecoacán,
Chiltepec); Campeche (Sonda de Campeche; Laguna de Términos, Puerto Real, Champotón, Campeche); Yucatán (Celestún, Sisal, Chuburna, Chelem, Yukalpetén, Progreso, Chicxulub, Telchac, Chavihau, Punta Arenas, Dzilam, Río Lagartos); Quintana Roo (Holbox).

Laevicardium mortoni $*$ (Conrad, 1830)

Distribución Geográfica.- BCnCñ. Cabo Cod a Florida; Alabama; Texas; Tamaulipas (Punta de Piedra, Barra del Tordo); Veracruz (Tecolutla); Tabasco (Complejo lagunar Carmen-Machona-Redonda, Complejo lagunar TupilcoOstión, Laguna de Mecoacán); Campeche (Sonda de Campeche, Laguna de Términos); Yucatán; Quintana Roo; Caribe.

Laevicardium serratum* (Linnaeus, 1758)

Distribución Geográfica.- CñpCnB. Virginia; De Carolina del Norte a ambos lados de Florida; Alabama; Texas; Tamaulipas (La Pesca, Barra de Ostiones, Tepehuajes, Barra del Tordo, Barra de Morón, Playa de Altamira, Playa Miramar); Veracruz (lado sur de la desembocadura del Arroyo 'Río Grande'; zona entre la desembocadura del Río Medio y Vergara, Vergara, Playa El Pulpo, Barra de Cazones, Bajo de Hornos); Campeche (Sonda de Campeche, arrecifes Arcas, Arenas); Yucatán (Arrecife Alacranes, Celestún, Chuburna, Yukalpeten, Dzilam); Quintana Roo (Holbox, Playa Carmen, y Arrecife Chinchorro); América Central; Bermudas; Caribe; Brasil.

Nemocardium tinctum (Dall, 1881)

Distribución Geográfica.- CñpCn. Sur de Florida; Alabama; Tamaulipas (Playa Bagdad, Barra El Mezquital, Barra Los Americanos, Barra de Catán, Barra Soto La Marina, La Pesca, Barra de Ostiones, Barra del Tordo, Lomas del Real, Playa Miramar); Campeche; hasta Brasil.

Papyridea semisulcata (Gray, 1825)

Distribución Geográfica.- Cñ. Sureste de Florida; Tamaulipas (Lomas del Real, Altamira; Playa Miramar, Madero, Tampico); Veracruz (de Pueblo Viejo a Tuxpan); Quintana Roo (Isla Mujeres y Playa Carmen); Bermudas; Caribe; Brasil.

Trachycardium isocardia* (Linnaeus, 1758)

Distribución Geográfica.- CñpCn. Texas; Tamaulipas (Carbonera, Punta de Piedra, Barra de Catán, La Pesca, Barra de Ostiones, Tepehuajes, Barra del Tordo, Barra de Morón, Playa de Altamira, Playa Miramar, Tampico); Veracruz (toda la costa); Tabasco (Complejo lagunar Carmen-Machona-Redonda, Complejo lagunar Tupilco- 
Ostión); Campeche (El Bosque, Laguna de Términos, Champotón, Campeche, arrecife Triángulos); Yucatán (Celestún, Sisal, Chuburna, Chelem, Yukalpetén, Progreso, Chicxulub, Telchac, Chavihau, Dzilam, Río Lagartos, Arrecife Alacranes, Punta Arenas); Quintana Roo (Holbox); Islas Bermudas; Caribe; Surinam; Sudamérica.

\section{FAMILIA VENERIDAE}

Agriopoma texasiana (Dall, 1892)

Distribución Geográfica.- CnpCñ. Florida; Texas; Tamaulipas (La Pesca, Tepehuajes).

\section{Anomalocardia auberiana (d'Orbigny, 1842)}

Distribución Geográfica.- CñpCnAg. Parte media oriental de Florida; Alabama; Texas; Tamaulipas (Punta de Piedra, Playa de Altamira, Tampico); Veracruz (toda la costa); Tabasco (Complejo lagunar Carmen-Machona-Redonda, Complejo lagunar Tupilco-Ostión, Chiltepec); Campeche (Laguna de Términos, El Bosque, Champotón, Cd. Sol, Seybaplaya); Yucatán (Celestún, Sisal, Chuburna, Chelem, Yukalpeten, Progreso, Chicxulub, Telchac); Quintana Roo (Holbox, Contoy, Isla Mujeres, Playa Carmen, Xcalac, arrecife Chinchorro, Cancún); Centroamérica; Caribe; Brasil; Uruguay.

Chione cancellata* (Linné, 1767)

Distribución Geográfica.- CñpCn. De Carolina del Norte a Florida; Alabama; Texas; Tamaulipas (Barra El Mezquital, Carbonera, Punta de Piedra, La Pesca, Tepehuajes, Barra de Ostiones, Barra del Tordo, Barra de Morón, Playa de Altamira, Playa Miramar, Tampico); Veracruz (toda la costa); Tabasco (Complejo lagunar Carmen-MachonaRedonda, Complejo lagunar Tupilco-Ostión, Laguna de Mecoacán, Chiltepec); Campeche (El Bosque, Sonda de Campeche, Laguna de Términos, Cd. Sol, Puerto Real, Champotón, Campeche, Seybaplaya); Yucatán (Arrecife Alacranes, Celestún, Sisal, Chuburna, Chelem, Yukalpetén, Progreso, Chicxulub, Telchac, Chavihau, Dzilam, Río Lagartos, Punta Arenas); Quintana Roo (Holbox, Contoy, Isla Mujeres, Playa Carmen, Xcalac, arrecifes Majahual y Chinchorro, Cancún); Costa Rica; Caribe; Brasil y sus islas oceánicas.

\section{Dosinia concentrica (Born, 1778)}

Distribución Geográfica.- CñpCn. Carolina del Sur; Florida; Texas; Tamaulipas (Playa Miramar, Tampico); Veracruz (de Pueblo Viejo a Tuxpan, y de Veracruz a Coatzacoalcos); Tabasco (Chiltepec); Campeche (Champotón, Campeche); Yucatán (Celestún); sur de Cuba a Brasil.
Dosinia discus* (Reeve, 1850)

Distribución Geográfica.- BCnCñ. De Virginia a Florida; Alabama; Texas; Tamaulipas (Barra de Catán, La Pesca, Barra de Ostiones, Tepehuajes, Barra del Tordo, Barra de Morón, Playa de Altamira, Playa Miramar); Veracruz (Playa El Pulpo, de Barra de Cazones a Coatzacoalcos); Campeche (Puerto Real, Champotón); Bahamas; Bermudas.

Dosinia elegans* Conrad, 1843

Distribución Geográfica.- CnCñ. Carolina del Norte; Alabama; Texas; Tamaulipas (La Pesca, Tepehuajes, Barra del Tordo); Veracruz (Playa El Pulpo, Barra de Cazones, Bajo de Hornos, de Veracruz a Coatzacoalcos); Tabasco (Chiltepec); Campeche (Laguna de Términos, El Bosque, Champotón, Campeche); Yucatán (Celestún, Chuburna, Yukalpetén, Progreso, Punta Arenas, Chicxulub, Telchac, Dzilam, Río Lagartos); Quintana Roo (Holbox); Caribe.

Globivenus rigida (Dillwyn, 1817)

Distribución Geográfica.- CñpCn. Cayos de Florida; Tamaulipas (Playa Bagdad, Punta de Piedra, Barra de Catán, La Pesca, Barra de Ostiones, Barra del Tordo); Veracruz (toda la costa); Tabasco (Chiltepec); Campeche (El Bosque, Sonda de Campeche, Puerto Real, Champotón); Caribe; Brasil.

Gouldia cerina (C.B. Adams, 1845)

Distribución Geográfica.- CñpCn. De Carolina del Norte a Florida; Tamaulipas (Barra El Mezquital, Barra Los Americanos, Barra de Catán, Barra Soto La Marina, Barra de Ostiones, Barra del Tordo, Lomas del Real, Playa Miramar, Tampico); Veracruz (toda la costa); Tabasco (Chiltepec); Campeche (El Bosque, Puerto Real, Champotón, Campeche); Bermudas; Caribe; Brasil.

Lirophora clenchi* Pulley, 1952

Distribución Geográfica.- CnCñ. Alabama; Texas; Tamaulipas (Playa Bagdad, Tepehuajes, Barra del Tordo, Playa de Altamira); Veracruz (toda la costa); Tabasco (Complejo lagunar Carmen-Machona-Redonda, Chiltepec); Campeche (El Bosque, Puerto Real, Champotón).

\section{Lirophora latilirata (Conrad, 1841)}

Distribución Geográfica.- CñpCn. De Carolina del Norte a Florida; Alabama; Texas; Tamaulipas (La Pesca); Veracruz (Laguna de Tamiahua, Playa El Pulpo, Barra de Cazones, Bajo de Hornos, Veracruz); Campeche (Champotón, Campeche); Brasil. 
Lirophora paphia Linnnaeus, 1767

Distribución Geográfica.- Cñ. Al sur de los Cayos de Florida; Tamaulipas (de Playa Bagdad a Tepehuajes y Barra del Tordo); Veracruz (toda la costa); Campeche (El Bosque, Puerto Real, Champotón); Caribe; Brasil.

Macrocallista maculata (Linnaeus, 1758)

Distribución Geográfica.- CñpCn. De Carolina del Norte al sur de Florida; Alabama; Texas; Tamaulipas (La Pesca, Barra de Ostiones, Tepehuajes, Barra de Morón, Tampico); Veracruz (toda la costa); Tabasco (Complejo lagunar Carmen- Machona- Redonda, Chiltepec); Campeche (Sonda de Campeche, Laguna de Términos; Campeche, Champotón); Yucatán (Celestún, Sisal, Chuburna, Chelem, Yukalpetén, Progreso, Chicxulub, Telchac, Chavihau, Dzilam, Río Lagartos, Arrecife Alacranes); Quintana Roo (Holbox); Bahamas; Bermudas; Caribe; Brasil.

Macrocallista nimbosa** (Ligthfoot, 1786)

Distribución Geográfica.- CnCñ. De Carolina del Norte a Florida; Texas; Tamaulipas (Barra de Catán); Veracruz (Punta Delgada, Punto Peñón, Veracruz, Alvarado); Tabasco; Campeche (El Bosque, Puero Real, Champotón).

Mercenaria campechiensis* (Gmelin, 1791)

Distribución Geográfica.- BCnCñ. Del sur de Nueva Jersey a Florida; Alabama; Texas; Tamaulipas (Playa Bagdad, Punta de Piedra, La Pesca, Barra de Ostiones, Tepehuajes, Barra del Tordo, Barra de Morón, Playa de Altamira, Playa Miramar, Tampico); Veracruz (desde Pueblo Viejo a Laguna Sontecomapan); Tabasco (Complejo lagunar Carmen-Machona-Redonda; Complejo Lagunar Tupilco-Ostión); Campeche (El Bosque, Laguna de Términos, Puerto Real, Champotón); Yucatán (Celestún, Sisal, Chelem, Yukalpeten, Progreso, Chicxulub, Telchac, Río Lagartos); Quintana Roo (Holbox); Cuba.

Petricolaria pholadiformis (Lamarck, 1818)

Distribución Geográfica.- BCnCñA. De Canadá (Golfo de San Lorenzo) a Florida; Alabama; Texas; Tamaulipas (La Pesca); Tabasco (Laguna de Mecoacán); Brasil; Uruguay.

Pitar dione (Linnaeus, 1758)

Distribución Geográfica.- CñpCn. Florida; Texas; Tamaulipas (Playa Miramar, Tampico); Veracruz (Cabo Rojo, Isla Lobos, Veracruz, Arrecife Blanquita, Isla Verde); Panamá; Caribe.
Puberella intapurpurea* (Conrad, 1849)

Distribución Geográfica.- CñpCnB. De Bahía de Chesapeake a Florida; Alabama; Texas; Tamaulipas (Playa Bagdad, La Pesca, Tepehuajes, Barra del Tordo, Barra de Morón, Playa de Altamira, Playa Miramar); Veracruz (Playa El Pulpo, Barra de Cazones, Cabo de Hornos); Tabasco (Complejo lagunar Carmen-Machona-Redonda, Complejo lagunar Tupilco-Ostión, Laguna de Mecoacán); Campeche (Sonda de Campeche, Puerto Real, Champotón); Yucatán; Caribe; Brasil.

Timoclea grus (Holmes, 1858)

Distribución Geográfica.- CnpCñ. De Carolina del Norte a Louisiana; Texas; Tamaulipas (Barra El Mezquital, Barra Los Americanos, Barra Soto La Marina, La Pesca, Barra de Ostiones, Barra del Tordo, Lomas del Real, Playa Miramar, Tampico); Veracruz (toda la costa); Tabasco (Complejo lagunar Carmen-Machona-Redonda); Campeche (El Bosque, Puerto Real, Champotón); Quintana Roo (Cabo Catoche, Playa Carmen).

\section{FAMILIA TELLINIDAE}

Angulus tampaensis (Conrad, 1866)

Distribución Geográfica.- CnCñ. Del sureste medio de Florida a Texas; Tamaulipas (Tepehuajes); Bahamas; Cuba.

Angulus texana Dall, 1900

Distribución Geográfica.- CnCñ. Desde Carolina del Norte al sur de Florida a Texas; Tamaulipas (Playa de Altamira, Tampico); Veracruz (toda la costa); Tabasco (Laguna del Carmen, Laguna Machona); Campeche (Puerto Real, Champotón, Sonda de Campeche); Bahamas; Cuba.

Eurytellina alternata alternata* (Say, 1822)

Distribución Geográfica.- CñpCn. Carolina del Norte a Florida; Alabama; Texas; Tamaulipas (Carbonera, Barra de Catán, La Pesca, Tepehuajes, Barra de Ostiones, Barra del Tordo, Playa de Altamira; Miramar); Veracruz (Laguna de la Mancha; Complejo lagunar Larga-Redonda o Mandinga Chica-Mandinga Grande, Playa El Pulpo, Barra de Cazones, Bajo de Hornos; Laguna Sontecomapan); Campeche (Sonda de Campeche, Laguna de Términos, Champotón); Yucatán (Celestún, Chuburna, Chelem, Yukalpetén, Progreso, Chicxulub, Río Lagartos, arrecife Alacranes); Quintana Roo (Holbox); Costa Rica; Caribe; Brasil. 
Eurytellina alternata tayloriana (Sowerby, 1867)

Distribución Geográfica.- CnCñ. Texas; Tamaulipas (La Pesca, Playa Miramar, Tampico); Veracruz (toda la costa); Tabasco (Laguna del Carmen, Laguna Machona, Chiltepec); Campeche (El Bosque, Puerto Real, Champotón, Campeche); Yucatán (Progreso, Celestún, Punta Arenas, El Cuyo); Quintana Roo (Isla Contoy).

Macoma tageliformis* Dall, 1900

Distribución Geográfica.- CñpCn. De Louisiana a Texas; Tamaulipas (La Pesca, Barra de Ostiones, Barra del Tordo, Tepehuajes, Barra de Morón, Playa de Altamira, Playa Miramar); Veracruz (Laguna Tampamachoco; Tuxpan, Laguna Sontecomapan), Tabasco (Complejo lagunar Carmen-Machona-Redonda; Complejo lagunar TupilcoOstión); Campeche (Sonda de Campeche); Yucatán (Celestún); Caribe; Surinam; Brasil.

Macoma tenta (Say, 1834)

Distribución Geográfica.- CñpCnB. Cabo Cod a sur de Florida; Alabama; Texas; Tamaulipas (Playa Bagdad, Barra de Catán, Barra Soto La Marina, La Pesca, Barra del Tordo); Veracruz (toda la costa); Tabasco (Complejo lagunar Carmen-Machona-Redonda, Chiltepec); Campeche (El Bosque, Puerto Real, Banco de Campeche, Laguna de Términos, Champotón, Campeche); Yucatán (Progreso, Punta Arenas, El Cuyo); Caribe; Brasil.

\section{Merisca aequistriata Say, 1824}

Distribución Geográfica.- CñpCn. De Carolina del Norte a Florida; Alabama; Texas; Tamaulipas (Barra El Mezquital, Barra Los Americanos, Barra de Catán, Barra Soto La Marina, La Pesca, Barra del Tordo, Lomas del Real, Tampico); Veracruz (toda la costa); Campeche (Banco de Campeche, El Bosque, Puerto Real, Champotón, Campeche); Yucatán (Progreso, Punta Arenas, El Cuyo); Quintana Roo (Cabo Catoche, Isla Contoy); Caribe; Brasil.

Strigilla mirabilis (Philippi, 1841)

Distribución Geográfica.- CñpCn. Alabama; Texas; Tamaulipas (Playa de Altamira); Tabasco (Complejo lagunar Carmen-Machona-Redonda); Campeche (Banco de Campeche); Yucatán (Arrecife Alacranes); Bermudas; Caribe; noreste de Brasil.

\section{Tellidora cristata (Récluz, 1842)}

Distribución Geográfica.- CnpCñ. Carolina del Norte; oeste y sur de Florida; Alabama; Texas; Tamaulipas (Playa de Altamira); Campeche (Laguna de Términos, Puerto Real, Champotón, Campeche); Yucatán (Celestún, Sisal,
Chuburna, Chelem, Yukalpetén, Progreso, Chicxulub); Quintana Roo (Holbox).

\section{FAMILIA DONACIDAE}

Donax variabilis* Say, 1822

Distribución Geográfica.- BCnCñ Nueva York, Virginia, Florida; Alabama; Texas; Tamaulipas (Playa Bagdad, Carbonera, Barra de Catán, La Pesca, Tepehuajes, Barra de Ostiones, Barra del Tordo, Barra de Morón, Playa de Altamira, Playa Miramar, Tampico); Veracruz (toda la costa); Tabasco (Complejo lagunar Carmen-MachonaRedonda, Chiltepec, Complejo Lagunar Tupilco-Ostión, Laguna de Mecoacán); Yucatán (Celestún, Progreso, Punta Arenas, El Cuyo).

Iphigenia brasiliensis (Lamarck, 1818)

Distribución Geográfica.- CñpCn. Sur de Florida; Texas; Tamaulipas (Carbonera, Punta de Piedra, La Pesca, Barra de Morón, Playa de Altamira; Playa Miramar); Tabasco (Complejo lagunar Carmen-Machona-Redonda); Caribe; Brasil.

\section{FAMILIA PSAMMOBIIDAE}

Asaphis deflorata (Linnaeus, 1758)

Distribución Geográfica.- Cñ. Sureste de Florida; Tamaulipas (Playa de Altamira, Tampico); Veracruz (toda la costa); Tabasco (Chiltepec); Campeche (Champotón); Yucatán (Celestún, Progreso); Quintana Roo (arrecife Chinchorro); Bermudas; Caribe; Brasil.

\section{FAMILIA SEMELIDAE}

Abra aequalis (Say, 1822)

Distribución Geográfica.- CñpCnB. De Delaware a Florida; Alabama; Texas; Tamaulipas (Playa Bagdad, Barra El Mezquital, Barra Los Americanos, Barra de Catán, Barra Soto La Marina, La Pesca, Barra de Ostiones, Barra del Tordo, Lomas del Real, Playa Miramar, Tampico); Veracruz (toda la costa); Tabasco (Laguna del Carmen, Laguna Machona, Chiltepec); Campeche (El Bosque, Puerto Real, Champotón, Campeche, Sonda de Campeche, Laguna de Términos); Yucatán (Progreso, Punta Arenas, El Cuyo); Quintana Roo (Cabo Catoche); Caribe; Surinam; Brasil.

\section{Semele bellastriata (Conrad, 1837)}

Distribución Geográfica.- CñpCn. De Carolina del Norte a Florida; Alabama; Texas; Tamaulipas (Playa Bagdad, Barra Soto La Marina, La Pesca, Barra del Tordo, Lomas del Real, Playa Miramar, Tampico); Veracruz (desde el norte a Roca Partida); Tabasco (Chiltepec); Campeche (El Bosque, 
Puerto Real; Banco de Campeche, Champotón); Yucatán; Bermudas, Caribe; Surinam; Brasil.

Semele proficua (Pulteney, 1799)

Distribución Geográfica.- CñpCnAg. Carolina del Norte; Florida; Alabama; Texas; Tamaulipas (Playa de Altamira); Veracruz (Laguna Tampamachoco; Bajo de Hornos; El Playón; Sistema Arrecifal del Puerto de Veracruz Laguna de la Mancha; Laguna Camaronera; Laguna Sontecomapan); Tabasco (Complejo lagunar CarmenMachona- Redonda); Yucatán; Centroamérica; Caribe; de la costa de Brasil hasta Argentina.

Semele purpurascens (Gmelin, 1791)

Distribución Geográfica.- CñpCnAg. De Carolina del Norte al sureste medio de Florida; Alabama; Texas; Tamaulipas (Tepehuajes); Tabasco; Campeche (El Bosque, Champotón y Campeche); Yucatán (Progreso); Centroamérica; Caribe; de la costa de Brasil a Uruguay.

Semelina nuculoides (Conrad, 1841)

Distribución Geográfica.- CnCñ. Carolina del Norte a sureste de Florida; Tamaulipas (Playa Miramar, Tampico); Veracruz (toda la costa); Tabasco (Laguna del Carmen, Chiltepec); Campeche (El Bosque, Puerto Real); Caribe.

\section{FAMILIA SOLECURTIDAE}

Solecurtus cumingianus (Dunker, 1861)

Distribución Geográfica.- CñpCn. Alabama; Texas; Tamaulipas (La Pesca, Tepehuajes, Playa de Altamira); Campeche (Banco de Campeche); Caribe; Colombia; Brasil.

\section{Tagelus divisus* (Spengler, 1794)}

Distribución Geográfica.- CñpCnB. Cabo Cod a sur de Florida; Alabama; Texas; Tamaulipas (Barra de Morón, Playa de Altamira, Tampico); Veracruz (toda la costa); Tabasco (Complejo lagunar Carmen-Machona-Redonda; Complejo lagunar Tupilco-Ostión); Campeche (Puerto Real); Yucatán (Yukalpetén); Quintana Roo; Bermudas, Caribe; Surinam; Brasil.

Tagelus plebeius* (Lightfoot, 1786)

Distribución Geográfica.- CñpCnAg. De Cabo Cod, Massachusetts a Florida; Alabama; Texas; Tamaulipas (La Pesca, Tepehuajes, Barra de Morón, Playa de Altamira, Playa Miramar, Tampico); Veracruz (Laguna Tampamachoco, Complejo lagunar Chica-Grande, Salado Chico, Complejo lagunar Larga-Redonda o Mandinga Chica-Mandinga Grande, Laguna de Alvarado, Laguna Sontecomapan,
Coatzacoalcos); Tabasco (Complejo lagunar CarmenMachona-Redonda, Complejo lagunar Tupilco-Ostión, Laguna de Mecoacán); Campeche (Puerto Real, Champotón, Laguna de Términos); Yucatán (Celestún, Yukalpetén, Chicxulub, Río Lagartos); Quintana Roo (Holbox); Caribe; Surinam; Brasil; Bahía Blanca en Argentina.

\section{FAMILIA MACTRIDAE}

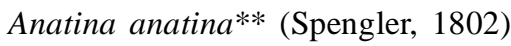

Distribución Geográfica.- CñpCn. De Carolina del Norte a Florida y Texas; Tamaulipas (Barra de Catán); Veracruz; Puerto Rico; Brasil.

Macrotoma fragilis* (Gmelin, 1791)

Distribución Geográfica.- CnCñ. De Carolina del Norte a Florida; Alabama; Texas; Tamaulipas (La Pesca, Barra del Tordo, Barra de Morón); Campeche (Sonda de Campeche); Yucatán (Celestún, Sisal, Chuburna, Yukalpetén, Progreso, Chicxulub, Telchac, Dzilam, Río Lagartos); Quintana Roo (Holbox); Caribe; Surinam.

Raeta plicatella* (Lamarck, 1818)

Distribución Geográfica.- CñpCnAg. De Virginia a Florida; Alabama; Texas; Tamaulipas (La Pesca, Barra del Tordo); Veracruz (centro y sur); Tabasco (Laguna del Carmen, Laguna Machona, Chiltepec); Campeche (El Bosque, Laguna de Términos, Champotón, Campeche, Banco de Campeche); Yucatán (Celestún, Progreso y Chicxulub); Caribe; Surinam; de Brasil a Argentina.

Rangianella flexuosa* (Conrad, 1839)

Distribución Geográfica.- CnCñ. De Florida a Texas; Tamaulipas (Playa Bagdad); Veracruz (Desde Pueblo Viejo a Laguna Sontecomapan); Tabasco (Laguna del Carmen, Laguna Machona); Campeche (Puerto Real, Laguna de Términos).

\section{FAMILIA CORBULIDAE}

Caryocorbula caribaea (D' Orbigny, 1853)

Distribución Geográfica.- BCnCñ. Massachusetts a Florida; Texas; Tamaulipas (Playa Bagdad, Barra El Mezquital, Barra Los Americanos, Barra de Catán, Barra Soto La Marina, La Pesca, Barra de Ostiones, Barra del Tordo, Lomas del Real, Playa Miramar, Tampico); Veracruz (toda la costa); Tabasco (Chiltepec); Campeche (El Bosque, Laguna de Términos, Puerto Real, Champotón, Campeche); Caribe. 
Caryocorbula contracta Say, 1822

Distribución Geográfica.- CñpCn. Cabo Cod a Florida; Tamaulipas (Playa Miramar, Tampico); Veracruz (toda la costa); Tabasco (Complejos lagunarios Carmen-MachonaRedonda y Tupilco-Ostión, Chiltepec); Campeche (El Bosque, Laguna de Términos, Puerto Real, Champotón); Caribe; Brasil.

Corbula barratiana C. B. Adams, 1852

Distribución Geográfica.- CñpCn. Carolina del Norte a Florida; Tamaulipas (Barra El Mezquital, Barra Soto La Marina, La Pesca, Barra de Ostiones, Barra del Tordo, Playa Miramar, Tampico); Veracruz (Pueblo Viejo a Punta del Morro, Coatzacoalcos); Tabasco (Laguna del CarmenMachona, Chiltepec); Campeche (El Bosque, Puerto Real, Champotón); Caribe; Surinam; Brasil.

Varicorbula krebsiana (C. B. Adams, 1852)

Distribución Geográfica.- CñCn. Carolina del Norte; Florida; Tamaulipas (Playa Bagdad, Barra El Mezquital, Barra Los Americanos, Barra de Catán, Barra Soto La Marina, La Pesca, Barra de Ostiones, Barra del Tordo, Lomas del Real, y Playa Miramar, Tampico); Veracruz (toda la costa); Tabasco (Lagunas del Carmen y Machona, Chiltepec); Campeche (El Bosque, Puerto Real, Champotón, Campeche); Yucatán (Progreso, Punta Arenas, El Cuyo); Caribe.

\section{FAMILIA PHOLADIDAE}

Cyrtopleura costata* (Linnaeus, 1758)

Distribución Geográfica.- CñpCnB. De Cabo Cod, Massachusetts a Nueva York; Florida; Alabama; Texas; Tamaulipas (La Pesca, Tepehuajes, Barra de Ostiones, Barra del Tordo, Barra de Morón, Playa de Altamira; Playa Miramar); Veracruz (Punta del Morro a Coatzacoalcos); Tabasco (Chiltepec, Complejo lagunar Tupilco-Ostión); Campeche (Laguna de Términos, El Bosque, Champotón, Campeche); Yucatán (Celestún); Caribe; Brasil.

Martesia cuneiformes (Say, 1822)

Distribución Geográfica.- CñpCn. De Carolina del Norte a Florida; Alabama; Texas; Tamaulipas (La Pesca); Tabasco (Complejo lagunar Carmen-Machona-Redonda; Complejo lagunar Tupilco-Ostión); Caribe; Brasil.

Martesia fragilis Verrill \& Bush, 1898

Distribución Geográfica.- CñpCnB. Virginia; Texas; Tamaulipas (Playa Miramar, Tampico); Veracruz (toda la costa); Tabasco (Laguna del Carmen, Chiltepec); Campeche (El Bosque, Puerto Real, Laguna de Términos, Chapotón); Yucatán (Celestún); Bermudas; Caribe a Brasil.

Pholas campechiensis* Gmelin, 1791

Distribución Geográfica.- CñpCnAg. Carolina del Norte; Alabama; Texas; Tamaulipas (La Pesca, Barra de Morón, Playa de Altamira, Playa Miramar); Veracruz (Punta del Morro a Coatzacoalcos); Tabasco (Chiltepec, Complejo lagunar Carmen-Machona-Redonda); Campeche (El Bosque, Champotón, Campeche); Yucatán (Celestún); Campeche (El Bosque, Champotón, Campeche); Yucatán (Celestún); Centroamérica; Caribe; Surinam; Brasil; Uruguay. 